\title{
Instance Optimal Decoding by Thresholding in Compressed Sensing
}

\author{
Albert Cohen, Wolfgang Dahmen, and Ronald DeVore*
}

November 1, 2008

\begin{abstract}
Compressed Sensing seeks to capture a discrete signal $x \in \mathbb{R}^{N}$ with a small number $n$ of linear measurements. The information captured about $x$ from such measurements is given by the vector $y=\Phi x \in \mathbb{R}^{n}$ where $\Phi$ is an $n \times N$ matrix. The best matrices, from the viewpoint of capturing sparse or compressible signals, are generated by random processes, e.g. their entries are given by i.i.d. Bernoulli or Gaussian random variables. The information $y$ holds about $x$ is extracted by a decoder $\Delta$ mapping $\mathbb{R}^{n}$ into $\mathbb{R}^{N}$. Typical decoders are based on $\ell_{1}$-minimization and greedy pursuit. The present paper studies the performance of decoders based on thresholding. For quite general random families of matrices $\Phi$, decoders $\Delta$ are constructed which are instance-optimal in probability by which we mean the following. If $x$ is any vector in $\mathbb{R}^{N}$, then with high probability applying $\Delta$ to $y=\Phi x$ gives a vector $\bar{x}:=\Delta(y)$ such that $\|x-\bar{x}\| \leq C_{0} \sigma_{k}(x)_{\ell_{2}}$ for all $k \leq a n / \log N$ provided $a$ is sufficiently small (depending on the probability of failure). Here $\sigma_{k}(x)_{\ell_{2}}$ is the error that results when $x$ is approximated by the $k$ sparse vector which equals $x$ in its $k$ largest coordinates and is otherwise zero. It is also shown that results of this type continue to hold even if the measurement vector $y$ is corrupted by additive noise: $y=\Phi x+e$ where $e$ is some noise vector. In this case $\sigma_{k}(x)_{\ell_{2}}$ is replaced by $\sigma_{k}(x)_{\ell_{2}}+\|e\|_{\ell_{2}}$.
\end{abstract}

AMS Subject Classification: 94A12, 65C99, 68P30, 41A25, 15A52

Key Words: Compressed sensing, best $k$-term approximation, instance optimal decoders, thresholding, noisy measurements, random matrices.

\section{Introduction}

\subsection{Background}

The typical paradigm for acquiring a compressed representation of a discrete signal $x \in$ $\mathbb{R}^{N}, N$ large, is to choose an appropriate basis, compute all of the coefficients of $x$ in this

*This research was supported by the Office of Naval Research Contracts ONR-N00014-08-1-1113, ONR N00014-05-1-0715; the ARO/DoD Contracts W911NF-05-1-0227 and W911NF-07-1-0185; the NSF Grant DMS-0810869; the Leibniz-Programme of DFG; and the French-German PROCOPE contract 11418YB 
basis, and then retain only the $k$ largest of these with $k<N$. Without loss of generality, we can assume that the appropriate basis is the canonical Kroenecker delta basis. If $S_{k} \subset\{1, \cdots, N\}$ denotes a set of indices corresponding to $k$ largest entries in $x$, then $x_{S_{k}}$ is the compressed approximation to $x$. Here and throughout this paper, for a set $T$ of indices, we denote by $x_{T}$ the vector which is identical to $x$ on $T$ but is zero outside $T$.

For any $\ell_{p}$ norm, this approximation process is equivalent to best $k$-term approximation. Namely, if

$$
\Sigma_{k}:=\left\{z \in \mathbb{R}^{N}: \#(\operatorname{supp}(z)) \leq k\right\},
$$

where $\operatorname{supp}(z)$ is the number of nonzero entries in $z$, and if for any norm $\|\cdot\|_{X}$ on $\mathbb{R}^{N}$, we define

$$
\sigma_{k}(x)_{X}:=\inf _{z \in \Sigma_{k}}\|x-z\|_{X}
$$

then $\left\|x-x_{S_{k}}\right\|_{\ell_{p}}=\left\|x_{S_{k}^{c}}\right\|_{\ell_{p}}=\sigma_{k}(x)_{\ell_{p}}$. That is, $x_{S_{k}}$ is a best approximation to $x$ from $\Sigma_{k}$. This approximation process should be considered as adaptive since the indices of those coefficients which are retained vary from one signal to another.

Since, in the end, we retain only $k$ entries of $x$ in the above compression paradigm, it seems wasteful to initially make $N$ measurements. The theory of compressed sensing as formulated by Candes, Romberg and Tao [8, 9] and by Donoho [14], asks whether it is possible to actually make a number $n$ of non-adaptive linear measurements, with $n$ comparable to $k$, and still retain the necessary information about $x$ in order to build a good compressed approximation. These measurements are represented by a vector

$$
y=\Phi x
$$

of dimension $n<N$ where $\Phi$ is an $n \times N$ measurement matrix (called a CS matrix). To extract the information that the measurement vector $y$ holds about $x$, one uses a decoder $\Delta$ which is a mapping from $\mathbb{R}^{n}$ into $\mathbb{R}^{N}$. The vector $x^{*}:=\Delta(y)=\Delta(\Phi x)$ is our approximation to $x$ extracted from the information $y$. In contrast to $\Phi$, the operator $\Delta$ is allowed to be non-linear.

In recent years, considerable progress has been made in understanding the performance of various choices of the measurement matrices $\Phi$ and decoders $\Delta$. Although not exclusively, by far most contributions focus on the ability of such an encoder-decoder pair $(\Phi, \Delta)$ to recover a sparse signal. For example, a typical theorem says that there are pairs $(\Phi, \Delta)$ such that whenever $x \in \Sigma_{k}$, with $k \leq$ an $/ \log (N / k)$, then $x^{*}=x$.

From both a theoretical and a practical perspective, it is highly desirable to have pairs $(\Phi, \Delta)$ that are robust in the sense that they are effective even when the vector $x$ is not assumed to be sparse. The question arises as to how we should measure the effectiveness of such an encoder-decoder pair $(\Phi, \Delta)$ for non-sparse vectors. In [6] we have proposed to measure such performance in a metric $\|\cdot\|_{X}$ by the largest value of $k$ for which

$$
\|x-\Delta(\Phi x)\|_{X} \leq C_{0} \sigma_{k}(x)_{X}, \quad \forall x \in \mathbb{R}^{N}
$$

with $C_{0}$ a constant independent of $k, n, N$. We say that a pair $(\Phi, \Delta)$ which satisfies property (1.4) is instance-optimal of order $k$ with constant $C_{0}$. It was shown that this measure of performance heavily depends on the norm employed to measure error. Let us illustrate this by two contrasting results from [6]: 
(i) If $\|\cdot\|_{X}$ is the $\ell_{1}$-norm, it is possible to build encoding-decoding pairs $(\Phi, \Delta)$ which are instance-optimal of order $k$ with a suitable constant $C_{0}$ whenever $n \geq$ $c k \log (N / k)$ provided $c$ and $C_{0}$ are sufficiently large. Moreover the decoder $\Delta$ can be taken as

$$
\Delta(y):=\underset{\Phi z=y}{\operatorname{argmin}}\|z\|_{\ell_{1}} .
$$

Therefore, in order to obtain the accuracy of $k$-term approximation, the number $n$ of non-adaptive measurements need only exceed the amount $k$ of adaptive measurements by the small factor $c \log (N / k)$. We shall speak of the range of $k$ which satisfy $k \leq a n / \log (N / k)$ as the large range since it is the largest range of $k$ for which instance-optimality can hold.

(ii) In the case $\|\cdot\|_{X}$ is the $\ell_{2}$-norm, if $(\Phi, \Delta)$ is any encoding-decoding pair which is instance-optimal of order $k=1$ with a fixed constant $C_{0}$, then the number of measurement $n$ is always larger than $a N$ where $a>0$ depends only on $C_{0}$. Therefore, the number of non-adaptive measurements has to be very large in order to compete with even one single adaptive measurement.

The matrices $\Phi$ which have the largest range of instance-optimality for $\ell_{1}$ are all given by stochastic constructions. Namely, one creates an appropriate random family $\Phi(\omega)$ of $n \times N$ matrices on a probability space $(\Omega, \rho)$ and then shows that with high probability on the draw, the resulting matrix $\Phi=\Phi(\omega)$ will satisfy instance-optimality for the large range of $k$. There are no known deterministic constructions. The situation is even worse in the sense that given an $n \times N$ matrix $\Phi$ there is no simple method for checking its range of instance-optimality.

While the above results show that instance-optimality is not a viable concept in $\ell_{2}$, it turns out that the situation is not as bleak as it seems. For example, a more optimistic result was established by Candes, Romberg and Tao in [9]. They show that if $n \geq$ $c k \log (N / k)$ it is possible to build pairs $(\Phi, \Delta)$ such that for all $x \in \mathbb{R}^{\mathrm{N}}$,

$$
\|x-\Delta(\Phi x)\|_{\ell_{2}} \leq C_{0} \frac{\sigma_{k}(x)_{\ell_{1}}}{\sqrt{k}},
$$

with the decoder again defined by (1.5). This implies in particular that $k$-sparse signals are exactly reconstructed and that signals $x$ in the space weak $\ell_{p}$ (denoted by $w \ell_{p}$ ) with $\|x\|_{w \ell_{p}} \leq M$ for some $p<1$ are reconstructed with accuracy $C_{0} M k^{-s}$ with $s=1 / p-1 / 2$. This bound is of the same order as the best estimate available on $\max \left\{\sigma_{k}(x)_{\ell_{2}}:\|x\|_{w \ell_{p}} \leq\right.$ $M\}$. Of course, this result still falls short of instance-optimality in $\ell_{2}$ as it must.

The starting point of the present paper is the intriguing fact, that instance-optimality can be attained in $\ell_{2}$ if one accepts a probabilistic statement. A first result in this direction, obtained by Cormode and Mutukrishnan in [7], shows how to construct random $n \times N$ matrices $\Phi(\omega)$ and a decoder $\Delta=\Delta(\omega), \omega \in \Omega$, such that for any $x \in \mathbb{R}^{N}$,

$$
\|x-\Delta(\Phi x)\|_{\ell_{2}} \leq C_{0} \sigma_{k}(x)_{\ell_{2}}
$$

holds with overwhelming probability (larger than $1-\epsilon(n)$ where $\epsilon(n)$ tends rapidly to 0 as $n \rightarrow+\infty)$ as long as $k \leq a n /(\log N)^{5 / 2}$ with $a$ suitably small. Note that this result 
says that given $x$, the set of $\omega \in \Omega$ for which (1.7) fails to hold has small measure. This set of failure will depend on $x$.

From our viewpoint, instance-optimality in probability is the proper formulation in $\ell_{2}$. Indeed, even in the more favorable setting of $\ell_{1}$, we can never put our hands on matrices $\Phi$ which have the large range of instance-optimality. We only know with high probability on the draw, in certain random constructions, that we can attain instance-optimality. So the situation in $\ell_{2}$ is not that much different from that in $\ell_{1}$.

The results in [6] pertaining to instance-optimality in probability asked two fundamental questions: (i) can we attain instance-optimality for the largest range of $k$, i.e. $k \leq a n / \log (N / k)$, and (ii) what are the properties of random families that are needed to attain this performance. We showed that instance-optimality can be obtained in the probabilistic setting for the largest range of $k$, i.e. $k \leq a n / \log (N / k)$ using quite general constructions of random matrices. Namely, we introduced two properties for a random matrix $\Phi$ which ensure instance-optimality in the above sense and then showed that these two properties hold for rather general constructions of random matrices (such as Gaussian and Bernoulli). However, one shortcoming of the results in [6] is that the decoder used in establishing instance-optimality was defined by minimizing $\|y-\Phi x\|_{\ell_{2}}$ over all $k$-sparse vectors, a task which cannot be achieved in any reasonable computational time.

\subsection{Objectives}

In the present paper, we shall be interested in which practical decoders can be used with a general random family so as to give a sensing system which has instance-optimality in probability for $\ell_{2}$ for the largest range of $k$. The first result in this direction was given by Wojtasczcek [24] who has shown that $\ell_{1}$-minimization can be used with Gaussian random matrices to attain instance-optimality for this large range of $k$. This result was recently generalized in [12] to arbitrary random families in which the entries of the matrix are generated by independent draws of a sub-gaussian random variables. This result includes Bernoulli matrices whose entries take the values $\pm 1 / \sqrt{n}$.

The problem of decoding in compressed sensing, as well as for more general inverse problems, is a very active area of research. In addition to $\ell_{1}$-minimization and its efficient implementation, several alternatives have been suggested as being possibly more efficient. These include decoding based on greedy procedures such as Orthogonal Matching Pursuit (OMP) (see [15, 20, 21, 22]) as well as decoding through weighted least squares [11]. Some of the pertinent issues in analyzing a decoding method is the efficiency of the method (number of computations) and the required storage needed.

Concerning efficiency, Gilbert and Tropp [15] have proposed to use a greedy procedure, known as Orthogonal Matching Pursuit (OMP) algorithm, in order to define $\Delta(y)$. The greedy algorithm identifies a set of $\Lambda$ of column indices which can be used to decode $y$. Taking zero as an initial guess, successive approximations to $y$ are formed by orthogonally projecting the measurement vector $y$ onto the span of certain incrementally selected columns $\phi_{j}$ of $\Phi$. In each step, the current set of columns is expanded by one further column that maximizes the modulus of the inner product with the current residual. The following striking result was proved in [15] for a probabilistic setting for general random matrices which include the Bernouli and Gaussian families: if $n \geq c k \log N$ with $c$ suffi- 
ciently large, then for any $k$ sparse vector $x$, the OMP algorithm returns exactly $x^{k}=x$ after $k$ iterations, with probability greater than $1-N^{-b}$ where $b$ can be made arbitrarily large by taking $c$ large enough.

Decoders like OMP are of high interest because of their efficiency. The above result of Gilbert and Tropp remains as the only general statement about OMP in the probabilistic setting. A significant breakthrough on decoding using greedy pursuit was given in the paper of Needel and Vershynin [20] (see also their followup [21]) where they showed the advantage of adjoining a batch of coordinates at each iteration rather than just one coordinate as in OMP. They show that such algorithms can deterministically capture sparse vectors for a slightly smaller range than the large range of $k$.

The present paper examines decoders based on thresholding and asks whether such algorithms can be used as decoders to yield $\ell_{2}$ instance-optimality in probability for general families of random matrices. We will describe in Section 6 a greedy thresholding scheme, referred to as $\mathbf{S T h r e s h}$, and prove that it gives instance-optimality in probability in $\ell_{2}$ for the large range of $k$. This algorithm adds a batch of coordinates at each iteration and then uses a thinning procedure to possibly remove some of them at later iterations. Conceptually, one thinks in terms of a bucket holding all of the coordinates to be used in the construction of $x$. In the analysis of such algorithms it is important to not allow more than a multiple of $k$ coordinates to gather in the bucket. The thinning is used for this purpose.

While preparing this paper, we became aware of the work of Needel and Tropp [22] in which they develop a deterministic algorithm (called COSAMP) which has features similar to ours. In fact, we have employed some of the ideas of that paper in our analysis. This will be discussed in more detail after we give a precise description of our algorithm.

While the benchmark of instance-optimality covers the case of an input signal $x$ which is a perturbation of a sparse signal, it is not quite appropriate for dealing with possible noise in the measurements. By this we mean that instead of measuring $\Phi x$, our measurement vector $y$ is of the form

$$
y=\Phi x+e,
$$

with $e \in \mathbb{R}^{n}$ a noise vector. SThresh will also perform well in this noisy setting. Stability under noisy measurements has been also established for COSAMP ([22]) as well as for schemes based on $\ell_{1}$-regularization [9]. While this latter strategy requires a-priori knowledge about the noise level, this is not the case for COSAMP and the schemes developed in this paper.

A brief overview of our paper is the following. In the next section, we introduce the probabilistic properties we will require of our random families. In $\S 3$, we introduce a deterministic algorithm based on thresholding and analyze its performance. This algorithm is then used as a basic step in the greedy decoding algorithm for stochastic families in the following section $\S 4$. In this section, we prove that the stochastic decoding algorithm gives instance optimality in probability. As we have noted above, a key step in this decoding is a thinning of the indices placed into the bucket. It is an intriguing question whether this thinning is actually necessary. This leads us to consider an algorithm without thinning. We introduce such an algorithm in $\S 6$ and we show in $\S 7$ that almost gives instanceoptimality in probability for $\ell_{2}$ for the large range of $k$. The results for that algorithm are weaker than the thinning algorithms in two ways. First they require the addition of 
a small term $\epsilon$ to $\sigma_{k}(x)_{\ell_{2}}$ and secondly the range of $k$ is slightly smaller than the large range. Finally, we append in $\S 8$ the proof that random matrices such as Gaussian and Bernoulli as well as uniform vectors on the unit sphere satisfy the properties which are used in the analysis of both algorithms.

While a lot of progress has been made on understanding the performance of greedy algorithms for decoding in compressed sensing, there remain fundamental unsettled questions. The most prominent is whether the original OMP algorithm can indeed give instance optimality in probability for $\ell_{2}$ for the large range of $k$.

\section{The Setting}

As we have already mentioned, one of our goals is to derive results that hold for general random families. In this section, we state general properties of random families which will be used as assumptions in our theorems.

We consider random $n \times N$ matrices $\Phi=\Phi(\omega)$, on a probability space $(\Omega, \rho)$. We denote the entries in $\Phi$ by $\phi_{i, j}, 1 \leq i \leq n, 1 \leq j \leq N$ and denote the $j$-th column of $\Phi$ by $\phi_{j}, j=1, \ldots, N$. One of the main properties needed of random families for compressed sensing is that given any $x \in \mathbb{R}^{\mathrm{N}}$, with high probability $\Phi x$ has norm comparable to that of $x$. We formulate this in

P1: For any $x \in \mathbb{R}^{\mathrm{N}}$ and $\delta>0$, there is a set $\Omega_{1}(x, \delta) \subset \Omega$ such that

$$
\left|\|\Phi x\|_{\ell_{2}}^{2}-\|x\|_{\ell_{2}}^{2}\right| \leq \delta\|x\|_{\ell_{2}}^{2}, \quad \omega \in \Omega_{1}(x, \delta),
$$

and

$$
\rho\left(\Omega_{1}^{c}(x, \delta)\right) \leq b_{1} e^{-c_{1} n \delta^{2}},
$$

where $b_{1}$ and $c_{1}$ are absolute constants.

An important consequence of property $\mathbf{P 1}$, often used in compressed sensing, is the following Restricted Isometry Property (RIP), as formulated by Candes and Tao [8]:

$\operatorname{RIP}(k, \eta): \quad$ An $n \times N$ matrix $\Phi_{0}$ is said to satisfy the Restricted Isometry Property of order $m$ with constant $\eta \in(0,1)$, if

$$
(1-\eta)\|x\|^{2} \leq\left\|\Phi_{0} x\right\|^{2} \leq(1+\eta)\|x\|^{2}, \quad x \in \Sigma_{m}
$$

It was shown in [3] that $\mathbf{P 1}$ implies RIP. More precisely, their analysis gives the following fact (which will also be proved in the Appendix §8).

Proposition 2.1 Whenever the random family $\Phi=\Phi(\omega), \omega \in \Omega$, of $n \times N$ matrices satisfies $\mathbf{P 1}$, then for each $\eta \in(0,1)$ there exists a subset $\Omega_{0}(m, \eta, \Phi) \subset \Omega$ with

$$
\rho\left(\Omega_{0}(m, \eta)^{c}\right) \leq b_{1} e^{-\frac{c_{1} n \eta^{2}}{4}+m[\log (e N / m)+\log (12 / \eta)]}
$$

where $b_{1}, c_{1}$ are the constants from $\mathbf{P} \mathbf{1}$, such that for each draw $\omega \in \Omega_{0}(m, \eta)$ the matrix $\Phi$ satisfies $\mathbf{R I P}(m, \eta)$ (order $m$ with constant $\eta)$. In particular, given $\eta$, if a is chosen suitably small (depending on $\eta$ ) then with high probability $\Phi$ will satisfy $\operatorname{RIP}(m, \eta)$ as long as $m \leq$ an $/ \log (N / m)$, i.e for the large range of $m$. 
Later we shall have to apply RIP to different groups of random matrivces $\Phi_{j}$. Including $\Phi$ as a parameter in sets of type $\Omega_{0}$ will indicate which group of matrices we refer to when invoking RIP.

\section{A deterministic thresholding algorithm}

In this section, we shall introduce a deterministic thresholding algorithm. Later, we shall embed this algorithm into the probabilistic setting and show that the corresponding probabilistic algorithm has $\ell_{2}$ instance optimality in probability.

We continue to denote by $k$ the envisaged range of instance optimality. We shall assume throughout this section that $\Phi$ is an $n \times N$ compressed sensing matrix that satisfies the $\mathbf{R I P}(m, \eta)$ where $m \geq 3 k$ is an integer which will be specified later. For the validity of the theorems that follow, there will also be a restriction that $\eta$ is sufficiently close to 0 .

\subsection{Description of the thresholding algorithm and main result}

In this section, we shall describe our thresholding algorithm. The algorithm starts with an input vector $y \in \mathbb{R}^{n}$ and generates a set $\Lambda$ of at most $k$ indices. The input vector $y$ is either $y=\Phi x$ in the noiseless case or $y=\Phi x+e$ in the presence of noise $e$ in the measurements. The output of the algorithm is a vector $x^{*}$ which is an approximation to $x$ determined by the noisy information $y$.

We now describe our thresholding algorithm for decoding an input vector $v \in \mathbb{R}^{n}$ of either type:

$\operatorname{DThresh}[v, k, \delta] \rightarrow x^{*}$

(i) Fix a thresholding parameter $\delta>0$. Choose the sparsity index $k$, let $r^{0}:=v$, $x^{0}:=0$, and set $j=0, \Lambda_{0}=\bar{\Lambda}_{0}=\emptyset$.

(ii) If $j=k$ stop and set $x^{*}:=x^{j}$.

(iii) Given $\Lambda_{j}$ calculate the residual $r^{j}:=v-\Phi x^{j}$ for the input vector $v$ and define

$$
\tilde{\Lambda}_{j+1}:=\left\{i \in\{1, \ldots, N\}:\left|\left\langle r^{j}, \phi_{i}\right\rangle\right| \geq \frac{\delta\left\|r^{j}\right\|}{\sqrt{k}}\right\}
$$

If $\tilde{\Lambda}_{j+1}=\emptyset$, stop and output $\Lambda^{*}=\Lambda_{j}$ and $x^{*}:=x^{j}$.

Otherwise set $\bar{\Lambda}_{j+1}:=\Lambda_{j} \cup \tilde{\Lambda}_{j+1}$.

(iv) Compute $\hat{x}\left(\bar{\Lambda}_{j+1}\right)$ (according to (5.13)) as

$$
\hat{x}\left(\bar{\Lambda}_{j+1}\right)=\operatorname{argmin}_{\operatorname{supp}(z) \subseteq \bar{\Lambda}_{j+1}}\|\Phi z-v\|,
$$

and define $\Lambda_{j+1}$ as the set indices $\nu \in \bar{\Lambda}_{j+1}$ corresponding to the $k$ largest (in absolute value) entries in $\hat{x}\left(\bar{\Lambda}_{j+1}\right)$. Let $x^{j+1}:=\hat{x}\left(\bar{\Lambda}_{j+1}\right)_{\Lambda_{j+1}}, j+1 \rightarrow j$ and return to (ii). 
Step (iii) is a thinning step which prevents the bucket of indices to get too large so that in our analysis $\mathbf{R I P}(\eta, m)$ will turn out to remain applicable for a fixed suitable multiple $m$ of $k$.

Perhaps a few remarks concerning a comparison with COSAMP are in order. In both schemes any a priori knowledge about the noise level is not needed but the envisaged sparsity range $k$ appears as a parameter in the scheme. This is in contrast to $\ell_{1}$-regularization in [9] which, however, does seem to require a priori knowledge about the noise level. Of course, one can take $k$ as the largest value for which the scheme can be shown to perform well. The subsequent analysis will show that this is indeed the case for the maximal range.

While DThresh as well as COSAMP are based on thresholding, COSAMP from the very beginning always works with least squares projections of size $2 k$. In the above scheme the sets of active indices $\Lambda_{j}$ are allowed to grow and, in fact, the scheme may terminate before they ever reach size $k$.

The following theorem summarizes the convergence properties of DThresh.

Theorem 3.1 Assume that $\delta, \eta \leq 1 / 32$ and that the matrix $\Phi$ satisfies $\operatorname{RIP}(m, \eta)$ with $m \geq\left\lceil k\left(1+\frac{3}{2 \delta^{2}}\right)\right\rceil$. Then for any $x \in \mathbb{R}^{N}$ and $y=\Phi x+e$ the output $x^{*}$ of $\mathbf{D T h r e s h}[y, k, \delta]$ has the following properties:

(i) If in addition $x \in \Sigma_{k}$, then the output $x^{*}$ satisfies

$$
\left\|x-x^{*}\right\| \leq 90\|e\|
$$

(ii) If $x \in \mathbb{R}^{N}$ and $x_{S_{k}}$ is its best approximation from $\Sigma_{k}$, i.e. the indices in $S_{k}$ identify the $k$ largest terms (in absolute value) in $x$, then

$$
\left\|x-x^{*}\right\| \leq 90\left[\left\|\Phi\left(x-x_{S_{k}}\right)\right\|+\|e\|\right]
$$

(iii) For arbitrary $x \in \mathbb{R}^{N}$, one has

$$
\left\|x-x^{*}\right\| \leq 90\left((1+\eta)^{1 / 2}\left(\frac{\sigma_{k}(x)_{\ell_{1}^{N}}}{\sqrt{k}}+\sigma_{k}(x)_{\ell_{2}^{N}}\right)+\|e\|\right) .
$$

We postpone the proof of Theorem 3.1 to Section 5 and explain first its ramifications in the stochastic setting.

\section{Thresholding in the stochastic setting}

Let us now assume that $\Phi(\omega), \omega \in \Omega$, is a random family of matrices which satisfy P1. As we have shown in Proposition 2.1, with high probability on the draw (see $(2.4)), \Phi(\omega)$ will satisfy $\operatorname{RIP}(m, \eta), m$ a fixed multiple of $k$, for the large range of $k$, with constant $a$ depending on that multiple and on $\eta$. We shall use the following stochastic version SThresh of the thresholding algorithm which differs from DThresh only in the initialization step (i).

$\operatorname{SThresh}[v, k, \delta] \rightarrow x^{*}$ 
(i) Fix a thresholding parameter $\delta>0$ and the sparsity index $k$. Given any signal $x \in \mathbb{R}^{N}$ take a random draw $\Phi=\Phi(\omega)$ and consider as input the measurement vector $v=\Phi x+e \in \mathbb{R}^{n}$ where $e$ is a noise vector. Let $r^{0}:=v$, and set $j=0$, $\Lambda_{0}=\bar{\Lambda}_{0}=\emptyset$.

(ii) If $j=k$ stop and set $x^{*}:=x^{j}$.

(iii) Given $\Lambda_{j}$ calculate the residual $r^{j}:=v-\Phi x^{j}$ for the input vector $v$ and define

$$
\tilde{\Lambda}_{j+1}:=\left\{i \in\{1, \ldots, N\}:\left|\left\langle r^{j}, \phi_{i}\right\rangle\right| \geq \frac{\delta\left\|r^{j}\right\|}{\sqrt{k}}\right\}
$$

If $\tilde{\Lambda}_{j+1}=\emptyset$, stop and output $\Lambda^{*}=\Lambda_{j}$ and $x^{*}:=x^{j}$.

Otherwise set $\bar{\Lambda}_{j+1}:=\Lambda_{j} \cup \tilde{\Lambda}_{j+1}$.

(iv) Compute $\hat{x}\left(\bar{\Lambda}_{j+1}\right)$ (according to $\left.(5.13)\right)$ as

$$
\hat{x}\left(\bar{\Lambda}_{j+1}\right)=\operatorname{argmin}_{\operatorname{supp}(z) \subseteq \bar{\Lambda}_{j+1}}\|\Phi z-v\|,
$$

and define $\Lambda_{j+1}$ as the set indices $\nu \in \bar{\Lambda}_{j+1}$ corresponding to the $k$ largest (in absolute value) entries in $\hat{x}\left(\bar{\Lambda}_{j+1}\right)$. Let $x^{j+1}:=\hat{x}\left(\bar{\Lambda}_{j+1}\right)_{\Lambda_{j+1}}, j+1 \rightarrow j$ and return to (ii).

Notice that the output $x^{*}=x^{*}(\omega)$ is stochastic. From the analysis of the previous section, we can deduce the following theorem.

Theorem 4.1 Assume that $\delta \leq 1 / 63$ in $\mathbf{S T h r e s h}$ and that the stochastic matrices $\Phi(\omega)$ have property $\mathbf{P 1}$. Then, for any $x \in \mathbb{R}^{N}$ there exists a subset $\Omega(x)$ of $\Omega$ with

$$
\rho\left(\Omega(x)^{c}\right) \leq 2 b_{1} e^{-c_{1} n / 8 \cdot 63^{2}},
$$

such that for any $\omega \in \Omega(x)$ and measurements of the form $y=\Phi(\omega) x+e$, with $e \in \mathbb{R}^{n}$ a noise vector, the output $x^{*}$ of $\mathbf{S T h r e s h}[y, \delta, k]$ satisfies

$$
\left\|x-x^{*}\right\| \leq C \sigma_{k}(x)+90\|e\|, \quad k \leq a n / \log (N / n)
$$

with $C \leq 92$ and a depending only on $\delta, c_{1}$ and the bound on $\eta$.

In particular, when $e=0$ this algorithm is instance-optimal in probability in $\ell_{2}$ for the large range of $k$.

Proof: Fixing $\eta=1 / 63$ and $m=\left\lceil\left(1+\frac{3}{2 \delta^{2}}\right) k\right\rceil$ we know by Proposition 2.1 that there exists a set $\Omega_{0} \subset \Omega$ such that for $\omega \in \Omega_{0}$ the matrix $\Phi=\Phi(\omega)$ satisfies $\operatorname{RIP}(m, 1 / 63)$ and

$$
\rho\left(\Omega_{0}^{c}\right) \leq b_{1} e^{-\frac{c_{1} n}{4 \cdot 63^{2}}+m[\log 756+\log (e N / m)]} .
$$

Thus, as long as $N \geq 756 m / e$ it suffices to have $2 m \log (e N / m) \leq c_{1} n / 8 \cdot 63^{2}$, to ensure that

$$
\rho\left(\Omega_{0}^{c}\right) \leq b_{1} e^{-\frac{c_{1} n}{8 \cdot 63^{2}}}, \quad \text { whenever } \quad k \leq a n / \log (N / k)
$$


provided $a$ is sufficiently large. Thus, we infer from Theorem 3.1 (ii) that

$$
\left\|x-x^{*}\right\| \leq 90\left(\left\|\Phi\left(x-x_{S_{k}}\right)\right\|+\|e\|\right)
$$

holds for every $\omega \in \Omega_{0}$. Now, by Property $\mathbf{P 1}$, there exists a subset $\Omega_{1}\left(x_{S_{k}}^{c}, 1 / 63\right)$ with complement

$$
\rho\left(\Omega_{1}\left(x_{S_{k}}^{c}, 1 / 63\right)^{c}\right) \leq b_{1} e^{-c_{1} n / 63^{2}},
$$

such that $\left\|\Phi\left(x-x_{S_{k}}\right)\right\| \leq 1.013\left\|x-x_{S_{k}}\right\|$ which ensures the validity of $(4.2)$ with $\Omega(x):=$ $\Omega_{0} \cap \Omega_{1}\left(x_{S_{k}}^{c}, 1 / 63\right)$.

\section{Proof of Theorem 3.1}

We begin by collecting a few prerequisites.

\subsection{Consequences of RIP}

Let us first record some simple results that follow from the $\operatorname{RIP}(m, \eta)$ assumption. Most of the results we state in this subsection can be found in [20] but we include their simple proofs for completeness of the present paper.

Lemma 5.1 For any $I \subset\{1, \ldots, N\}$ with $\#(I) \leq m$ we have

$$
\left\|\Phi_{I}^{*}\right\|^{2}=\left\|\Phi_{I}\right\|^{2} \leq(1+\eta) .
$$

Proof: The equality in (5.1) holds because the norm of a matrix and its conjugate transpose are identical (this follows for example from the fact that $\|A\|=\sup _{\|x\|=1,\|y\|=1} y^{t} A x$ ). The upper inequality follows from the $\operatorname{RIP}(m, \eta)$ assumption because for any $x \in \mathbb{R}^{N}$, supported in $I$ one has $\left\|\Phi_{I} x\right\|=\left\|\Phi x_{I}\right\| \leq(1+\eta)^{1 / 2}\left\|x_{I}\right\|=(1+\eta)^{1 / 2}\|x\|$.

Lemma 5.2 For any $I$ with $\#(I) \leq m$ we have

$$
(1-\eta) \leq\left\|\Phi_{I}^{*} \Phi_{I} x\right\| \leq(1+\eta), \quad\left\|x_{I}\right\|=1 .
$$

and therefore

$$
\left\|\Phi_{I}^{*} \Phi_{I}-I d_{I}\right\| \leq \eta
$$

where $I d_{I}$ denotes the identity matrix of size \#(I).

Proof: The upper inequality in (5.2) follows from Lemma 5.1 and the lower inequality follows from $\operatorname{RIP}(m, \eta)$ since

$$
\left\|x_{I}\right\| \cdot\left\|\Phi_{I}^{*} \Phi_{I} x_{I}\right\| \geq x_{I}^{*} \Phi_{I}^{*} \Phi_{I} x_{I}=\left\|\Phi_{I} x_{I}\right\|^{2} \geq(1-\eta) .
$$

Hence all eigenvalues of $\Phi_{I}^{*} \Phi_{I}$ belong to $(1-\eta, 1+\eta)$. Thus the symmetric matrix $\Phi_{I}^{*} \Phi_{I}-I d_{I}$ has its eigenvalues in $(-\eta, \eta)$ which confirms $(5.3)$. 
Lemma 5.3 For any $I$ with $\#(I) \leq m$ and any $x$ with $\operatorname{supp}(x) \subseteq I$, we have

$$
\frac{(1-\eta)}{\sqrt{1+\eta}}\left\|\Phi_{I} x\right\| \leq(1-\eta)\|x\| \leq\left\|\Phi_{I}^{*} \Phi_{I} x\right\| \leq \sqrt{1+\eta}\left\|\Phi_{I} x\right\| .
$$

Proof: The upper inequality in (5.4) follows from Lemma 5.1. The two lower inequalities, follow from (2.3) and (5.2), respectively.

Lemma 5.4 Suppose that $T$ and $J$ are sets of indices such that $\#(J \cup T) \leq m$ and $J \cap T=\emptyset$. If $\operatorname{supp}(x)=T$ one has

$$
\left\|\Phi_{J}^{*} \Phi x\right\| \leq \eta\|x\| .
$$

Proof: Let $I:=J \cup T$. We extend the matrices $\Phi_{J}^{*}, \Phi_{T}^{*}$ to size $\#(I) \times n$ by adjoining rows that are identically zero when the indices are in $I \backslash J$ and $I \backslash T$ respectively. Similarly extend $\Phi_{T}$ so that it has columns indexed on $I$. Then, since $x$ is supported on $T \subset I$, we have

$$
\Phi_{J}^{*} \Phi x=\left[\Phi_{I}^{*} \Phi_{I}-\Phi_{T}^{*} \Phi_{T}\right] x=\left[\Phi_{I}^{*} \Phi_{I}-I d_{I}-\left(\Phi_{T}^{*} \Phi_{T}-I d_{I}\right)\right] x .
$$

Since the vectors $\left[\Phi_{I}^{*} \Phi_{I}-I d_{I}\right] x$ and $\left.\left[\Phi_{T}^{*} \Phi_{T}-I d_{I}\right)\right] x$ agree in all coordinates for which the latter vector is nonzero, we can take norms in (5.6), use Lemma 5.2 and obtain

$$
\left\|\Phi_{J}^{*} \Phi x\right\| \leq\left\|\left[\Phi_{I}^{*} \Phi_{I}-I d_{I}\right] x\right\| \leq \eta\|x\|,
$$

as desired.

As a summary of these results, under the assumption $\operatorname{RIP}(m, \eta)$, we have for any two disjoint sets $\Lambda, \Lambda^{\prime} \subset\{1, \ldots, N\}$ such that $\#\left(\Lambda \cup \Lambda^{\prime}\right) \leq m$, and for any vectors $u \in \mathbb{R}^{\#(\Lambda)}$, $v \in \mathbb{R}^{n}$, we have

$$
\left\|\Phi_{\Lambda^{\prime}} \Phi_{\Lambda} u\right\| \leq \eta\|u\| .
$$

Moreover, for any $\Lambda \subset\{1, \ldots, N\}$, \# $\Lambda \leq m$, one has

$$
\begin{aligned}
\left\|\Phi_{\Lambda}^{*} v\right\| & \leq(1+\eta)^{1 / 2}\|v\| \\
\left\|\Phi_{\Lambda}^{*} \Phi_{\Lambda} u\right\| & \leq(1 \pm \eta)\|u\| \\
\left\|\left(\Phi_{\Lambda}^{*} \Phi_{\Lambda}\right)^{-1} u\right\| & \doteq(1 \pm \eta)^{-1}\|u\| .
\end{aligned}
$$

We conclude this section with some remarks on solving least squares problems. Suppose that $\Phi$ satisfies $\operatorname{RIP}(m, \eta)$ for some $\eta<1$. Given any set $\Lambda \subset\{1, \ldots, N\}$ with cardinality $\leq m$ and any input vector $v \in \mathbb{R}^{n}$, the least squares problem

$$
\hat{u}(\Lambda):=\underset{\operatorname{supp}(z) \subseteq \Lambda}{\operatorname{argmin}}\|v-\Phi z\|
$$

has a unique solution given by the Moore-Penrose pseudo inverse

$$
\hat{u}(\Lambda)=\left[\Phi_{\Lambda}^{*} \Phi_{\Lambda}\right]^{-1} \Phi_{\Lambda}^{*} v .
$$

By (5.10) the solution can be computed in a stable way.

Notice that $\Phi_{\Lambda} \hat{u}(\Lambda)=P_{\Lambda} v$ where $P_{\Lambda}$ is the projector onto the span of the columns $\phi_{\nu}$, $\nu \in \Lambda$. 


\subsection{Analysis of DThresh}

In this section, we shall analyze the performance of the thresholding algorithm in the deterministic setting and prove that the output $x^{*}$ is a good approximation to $x$. We fix the threshold $\delta>0$ and assume that $\Phi$ satisfies the $\operatorname{RIP}(m, \eta)$ for some integer $m>\left(1+\frac{3}{2 \delta^{2}}\right) k$ and some constant $\eta<1$. We shall see that, if $\eta$ and $\delta$ are chosen sufficiently small, the scheme DThresh will have good convergence properties.

For our first lemma, we analyze thresholding when the input vector is $v=\Phi u+e$ with $u \in \Sigma_{2 k}$. Let $T$ denote the support of $u$ so that by assumption $\#(T) \leq 2 k$ and let $\Lambda(v, k)$ denote the set of coordinates $\nu$ with

$$
\left|\left\langle v, \phi_{\nu}\right\rangle\right| \geq \frac{\delta\|u\|}{\sqrt{k}} .
$$

Lemma 5.5 The set $\Lambda(v, k)$ contains at most $\frac{3 k}{2 \delta^{2}}$ coordinates.

Proof: Suppose $\Lambda(v, k)$ contains a set $I$ of $\leq m$ coordinates. Then from the definition of $\Lambda(v, k)$ and (5.9), we have

$$
\frac{\#(I) \delta^{2}\|v\|^{2}}{k} \leq\left\|\Phi_{I}^{*} v\right\|^{2} \leq(1+\eta)\|v\|^{2} \leq 3 / 2\|v\|^{2} .
$$

It follows that $\#(I) \leq \frac{3 k}{2 \delta^{2}}$ which proves the lemma.

The following lemma will be key to our error analysis.

Lemma 5.6 Assume that $v=y=\Phi x+e$ with $x \in \Sigma_{k}$ and that the threshold $\delta$ in $\operatorname{DThresh}[y, \delta, k]$ satisfies $\delta \leq 1 / 63$. Moreover, assume that $\Phi$ satisfies $\operatorname{RIP}(m, \eta)$ for a fixed $\eta \leq 1 / 63$ and $m>\left(1+3 / \delta^{2}\right) k$. Then for the iterates $x^{j}, j=0,1, \ldots$, produced by DThresh $[y, \delta, k]$ one has

$$
\left\|x-x^{j+1}\right\| \leq \frac{18}{61}\left\|x-x^{j}\right\|+\frac{144}{61}\|e\|,
$$

and

$$
\left\|x-x_{\Lambda_{j+1}}\right\| \leq \frac{3}{5}\left\|x-x_{\Lambda_{j}}\right\|+4\|e\|
$$

Proof: Let $S$ be the support of $x$. We fix $j$ and use the abbreviated notation $\bar{\Lambda}:=\bar{\Lambda}_{j+1}$ and $\hat{x}:=\hat{x}(\bar{\Lambda})$. Let $T:=S \cup \bar{\Lambda}$ which contains the support of $x-\hat{x}$. We have

$$
\begin{aligned}
\|x-\hat{x}\| & \leq(1-\eta)^{-1}\left\|\Phi_{T}^{*} \Phi_{T}(x-\hat{x})\right\| \\
& \leq(1-\eta)^{-1}\left\{\left\|\Phi_{T}^{*}\left[\Phi_{T}(x-\hat{x})+e\right]\right\|+\left\|\Phi_{T}^{*} e\right\|\right\} \\
& \leq(1-\eta)^{-1}\left\{\left\|\Phi_{S \backslash \bar{\Lambda}}^{*}\left[\Phi_{T}(x-\hat{x})+e\right]\right\|+(1+\eta)^{1 / 2}\|e\|\right\}
\end{aligned}
$$

where the first inequality uses (5.10) (which is applicable since the cardinality of $T$ is $\leq m$ because of Lemma 5.5), and the third inequality uses (5.1) and the fact that the inner product of

$$
\left[\Phi_{T}(x-\hat{x})+e\right]=y-\Phi_{\bar{\Lambda}} \hat{x}=y-P_{\bar{\Lambda}} y
$$


with any column of $\Phi$ with index inside $\bar{\Lambda}$ is zero.

We estimate the first term in (5.18) as follows

$$
\begin{aligned}
\left\|\Phi_{S \backslash \bar{\Lambda}}^{*}\left[\Phi_{T}(x-\hat{x})+e\right]\right\| & =\left\|\Phi_{S \backslash \bar{\Lambda}}^{*}\left(y-\Phi_{\bar{\Lambda}} \hat{x}\right)\right\| \\
& \leq\left\|\Phi_{S \backslash \bar{\Lambda}}^{*}\left[y-\Phi x^{j}\right]\right\|+\|\| \Phi_{S \backslash \bar{\Lambda}}^{*} \Phi\left(x^{j}-\hat{x}\right) \| \\
& =\left\|\Phi_{S \backslash \bar{\Lambda}}^{*}\left[r^{j}\right]\right\|+\left\|\Phi_{S \backslash \bar{\Lambda}}^{*}\left[\Phi x^{j}-\Phi \hat{x}\right]\right\| .
\end{aligned}
$$

To estimate the first term on the right side of (5.19), we use the fact that each inner product of $\phi_{\nu}, \nu \in S \backslash \bar{\Lambda}$, with $r^{j}$ is $\leq \delta / \sqrt{k}$ because of the definition of $\bar{\Lambda}$. Since $\#(S) \leq k$, using (5.1), we obtain

$$
\left\|\Phi_{S \backslash \bar{\Lambda}}^{*}\left[r^{j}\right]\right\| \leq \sqrt{\delta}\left\|r^{j}\right\|=\sqrt{\delta}\left\|\Phi\left(x-\hat{x}_{\Lambda_{j}}\right)+e\right\| \leq \sqrt{\delta}(1+\eta)^{1 / 2}\left\|x-x^{j}\right\|+\sqrt{\delta}\|e\| .
$$

For the second term on the right side of (5.19), we note that $\bar{\Lambda}$ is disjoint from $S \backslash \bar{\Lambda}$ and that $\Lambda_{j}=\operatorname{supp} x^{j} \subseteq \bar{\Lambda}$, so we can invoke (5.8) and obtain

$$
\left\|\Phi_{S \backslash \bar{\Lambda}}^{*}\left[\Phi x^{j}-\Phi \hat{x}\right] \leq \eta\right\| x^{j}-\hat{x} \| \leq \eta\left[\left\|x-x^{j}\right\|+\|x-\hat{x}\|\right] .
$$

If we use now the estimate (5.20) and (5.21) in (5.19), we obtain

$$
\left\|\Phi_{S \backslash \bar{\Lambda}}^{*}\left[\Phi_{T}(x-\hat{x})+e\right]\right\| \leq \sqrt{\delta}(1+\eta)^{1 / 2}\left\|x-x^{j}\right\|+\sqrt{\delta}\|e\|+\eta\left[\left\|x-x^{j}\right\|+\|x-\hat{x}\|\right]
$$

We now insert the latter estimate in (5.18) and obtain

$$
\|x-\hat{x}\| \leq(1-\eta)^{-1}\left(\eta\|x-\hat{x}\|+\left((1+\eta)^{1 / 2} \sqrt{\delta}+\eta\right)\left\|x-x^{j}\right\|+\left[\sqrt{\delta}+(1+\eta)^{1 / 2}\right]\|e\|\right) .
$$

We now bring the term involving $\|x-\hat{x}\|$ on the right to the left side and obtain

$$
\|x-\hat{x}\| \leq \frac{(1+\eta)^{1 / 2} \sqrt{\delta}+\eta}{(1-2 \eta)}\left\|x-x^{j}\right\|+\frac{\sqrt{\delta}+(1+\eta)^{1 / 2}}{(1-2 \eta)}\|e\| .
$$

Recalling that $\hat{x}=\hat{x}\left(\bar{\Lambda}_{j+1}\right)$ and that $x^{j+1}=\hat{x}\left(\bar{\Lambda}_{j+1}\right)_{\Lambda_{j+1}}$ is its best $k$-term approximation, we find

$$
\left\|x-x^{j+1}\right\| \leq\left\|x-\hat{x}\left(\bar{\Lambda}_{j+1}\right)\right\|+\left\|\hat{x}\left(\bar{\Lambda}_{j+1}\right)-\hat{x}\left(\bar{\Lambda}_{j+1}\right)_{\Lambda_{j+1}}\right\| \leq 2\left\|x-\hat{x}\left(\bar{\Lambda}_{j+1}\right)\right\|,
$$

since the support of $x$ has also size at most $k$. Thus we deduce from (5.24) and (5.25) that

$$
\left\|x-x^{j+1}\right\| \leq \frac{2\left((1+\eta)^{1 / 2} \sqrt{\delta}+\eta\right)}{(1-2 \eta)}\left\|x-x^{j}\right\|+\frac{2\left(\sqrt{\delta}+(1+\eta)^{1 / 2}\right)}{(1-2 \eta)}\|e\| .
$$

When we invoke our restrictions that both $\delta$ and $\eta$ are $\leq 1 / 63$, we arrive at (5.16).

To derive (5.17), we note that from (5.16) we obtain

$$
\left\|x-x_{\Lambda_{j+1}}\right\| \leq\left\|x-x^{j+1}\right\| \leq \frac{18}{61}\left\|x-x^{j}\right\|+\frac{144}{61}\|e\| .
$$


Since by Lemma $5.5 \#\left(S \cup \bar{\Lambda}_{j}\right) \leq k+\frac{3}{2 \delta^{2}} \leq m$, we can apply $\operatorname{RIP}(m, \eta)$ to conclude that

$$
\begin{aligned}
\left\|x-\hat{x}\left(\bar{\Lambda}_{j}\right)\right\| & \leq(1-\eta)^{-1 / 2}\left\|\Phi\left(x-\hat{x}\left(\bar{\Lambda}_{j}\right)\right)\right\| \leq(1-\eta)^{-1 / 2}\left[\left\|\Phi\left(x-\hat{x}\left(\bar{\Lambda}_{j}\right)\right)+e\right\|+\|e\|\right] \\
& =(1-\eta)^{-1 / 2}\left(\left\|y-P_{\bar{\Lambda}_{j}} y\right\|+\|e\|\right) \\
& \leq(1-\eta)^{-1 / 2}\left[\left\|y-\Phi x_{\Lambda_{j}}\right\|+\|e\|\right] \leq(1-\eta)^{-1 / 2}\left[\left\|\Phi\left(x-x_{\Lambda_{j}}\right)\right\|+2\|e\|\right] \\
& \leq(1-\eta)^{-1 / 2}\left[(1+\eta)^{1 / 2}\left\|x-x_{\Lambda_{j}}\right\|+2\|e\|\right] .
\end{aligned}
$$

Since $x^{j}$ is the best $k$-term approximation of $\hat{x}\left(\bar{\Lambda}_{j}\right)$ we can use (5.25) again, to conclude that

$$
\left\|x-x^{j}\right\| \leq 2\left(\frac{1+\eta}{1-\eta}\right)^{1 / 2}\left\|x-x_{\Lambda_{j}}\right\|+4(1-\eta)^{-1 / 2}\|e\| .
$$

Placing this in (5.27) and using the restriction $\eta \leq 1 / 63$ gives

$$
\left\|x-x_{\Lambda_{j+1}}\right\| \leq \frac{3}{5}\left\|x-x_{\Lambda_{j}}\right\|+\frac{217}{61}\|e\|
$$

and hence (5.17).

We can derive from this lemma several results about the convergence of DThresh. For this, we shall use the following lemma.

Lemma 5.7 Suppose that $x \in \mathbb{R}^{N}$ and $\alpha \leq 1 / \sqrt{2}$. Let $\Lambda_{0}=\emptyset$ and suppose $\Lambda_{j} \subset$ $\{1, \ldots, N\}, j=1,2, \ldots, j_{0}$, are sets such that

$$
\left\|x-x_{\Lambda_{j+1}}\right\| \leq \alpha\left\|x-x_{\Lambda_{j}}\right\|, \quad j=0, \ldots, j_{0}-1
$$

Then,

$$
\left\|x-x_{\Lambda_{j}}\right\| \leq \sigma_{j}(x), \quad j=0, \ldots, j_{0} .
$$

Proof: We prove this by induction on $j$. This is obviously true for $j=0$ and we now assume this is true for any $j<j_{0}$ and advance the induction hypothesis. Without loss of generality we can assume that $\left|x_{1}\right| \geq\left|x_{2}\right| \geq \ldots\left|x_{N}\right|$. If $\sigma_{j+1}(x) \geq \alpha \sigma_{j}(x)$, then

$$
\left\|x-x_{\Lambda_{j+1}}\right\| \leq \alpha\left\|x-x_{\Lambda_{j}}\right\| \leq \alpha \sigma_{j}(x) \leq \sigma_{j+1}(x) .
$$

On the other hand, if $\sigma_{j+1}(x)<\alpha \sigma_{j}(x)$, then $\sigma_{j}(x)^{2}-\left|x_{j+1}\right|^{2}<\alpha^{2} \sigma_{j}(x)^{2}$ or, in other words $\left|x_{j+1}\right|^{2}>\left(1-\alpha^{2}\right) \sigma_{j}(x)^{2}$. Now, by our induction assumption,

$$
\sum_{\nu \notin \Lambda_{j+1}} x_{\nu}^{2} \leq \alpha^{2} \sigma_{j}(x)^{2} \leq\left(1-\alpha^{2}\right) \sigma_{j}(x)^{2},
$$

because $\alpha^{2} \leq 1 / 2$. It follows that $\Lambda_{j+1}$ must contain every $i \leq j+1$ and so we again have (5.31).

We are now ready to complete the proof of Theorem 3.1, 
Proof of (i): We want to show that $\left\|x-x^{*}\right\| \leq A\|e\|$ with $A \leq 280$. Let $j_{0}$ be the terminating index of the algorithm. Suppose for some $j_{1} \leq j_{0}$ we have $\left\|x-x^{j_{1}}\right\| \leq A\|e\|$, for some $A$. From (5.16) we have by induction for any $j_{1} \leq j<j_{0}$ that

$$
\left\|x-x^{j+1}\right\| \leq \frac{18}{61}\left\|x-x^{j}\right\|+\frac{144}{61}\|e\| \leq A\|e\|
$$

whenever $A \geq \frac{162}{61}$, as desired.

For the next case, we assume that the algorithm terminates for some $j<k$, so that $\tilde{\Lambda}_{j}=\emptyset$ and hence $\Lambda_{j}=\Lambda_{j-1}$ and $x^{*}=x^{j}=x^{j-1}$. In this case, (5.16) gives that

$$
\left\|x-x^{*}\right\|=\left\|x-x^{j}\right\| \leq \frac{18}{61}\left\|x-x^{j-1}\right\|+\frac{144}{61}\|e\|=\frac{18}{61}\left\|x-x^{j}\right\|+\frac{144}{61}\|e\| .
$$

Thus, $\left\|x-x^{*}\right\| \leq A\|e\|$, as long as $A \geq \frac{144}{43}$, and we have proved this case as well.

The last possibility is that $\left\|x-x^{j}\right\| \geq A\|e\|$ for all $0 \leq j \leq k$. From (5.29), it follows that

$$
\left\|x-x_{\Lambda_{j}}\right\| \geq \frac{1}{2}\left(\frac{1-\eta}{1+\eta}\right)^{1 / 2}\left(\left\|x-x^{j}\right\|-\frac{4}{(1-\eta)^{1 / 2}}\|e\|\right)
$$

which, under the assumption that $\left\|x-x^{j}\right\| \geq A\|e\|$, yields

$$
\|e\| \leq \frac{2(1+\eta)^{1 / 2}}{(1-\eta)^{1 / 2} A-4}\left\|x-x_{\Lambda_{j}}\right\| .
$$

This together with (5.17) yields

$$
\left\|x-x_{\Lambda_{j+1}}\right\| \leq\left(.6+\frac{8(1+\eta)^{1 / 2}}{(1-\eta)^{1 / 2} A-4}\right)\left\|x-x_{\Lambda_{j}}\right\|, \quad 0 \leq j \leq k .
$$

One can check that as long as $A \geq 90$ the expression in parentheses on the right hand side of $(5.38)$ is less than $0.7 \leq 1 / \sqrt{2}$. We are then allowed to employ Lemma 5.7 to find

$$
\left\|x-x_{\Lambda_{k}}\right\| \leq \sigma_{k}(x)=0 .
$$

Using this in (5.29) gives $\left\|x-x^{*}\right\|=\left\|x-x^{k}\right\| \leq 4(1-\eta)^{-1 / 2}\|e\|<A\|e\|$ which concludes the proof of (i).

Proof of (ii): For an arbitrary signal $x \in \mathbb{R}^{N}$, we let $S$ be the set of $k$ indices corresponding to the $k$ largest entries (in absolute value) of $x$ and set

$$
y=\Phi x+e=\Phi x_{S}+\Phi x_{S^{c}}+e=: \Phi x_{S}+\tilde{e}
$$

with $\tilde{e}:=e+\Phi x_{S^{c}}$. Applying (i), we have

$$
\left\|x-x^{*}\right\| \leq 90\|\tilde{e}\| \leq 90\left(\left\|\Phi x_{S^{c}}\right\|+\|e\|\right)
$$

which proves (ii). 
Proof of (iii): Again, let $S$ be a set of coordinates corresponding to the $k$ largest entries in $x$. From $\operatorname{RIP}(m, \eta)$ one deduces that

$$
\left\|\Phi x_{S^{c}}\right\| \leq(1+\eta)^{1 / 2}\left(\frac{\sigma_{k}(x)_{\ell_{1}}}{\sqrt{k}}+\sigma_{k}(x)_{\ell_{2}}\right) .
$$

For the proof of this see either [9] or [6]. For convenience of the reader we sketch it here. Let $T_{0}:=S$ and $T_{i+1}$ denote the set of indices corresponding to the next $k$ largest (in absolute value) entries of $x_{\left(T_{0} \cup \cdots \cup T_{i}\right)^{c}}$ so that $\left\|x_{T_{i+1}}\right\| \leq k^{-1 / 2}\left\|x_{T_{i}}\right\|_{\ell_{1}}$. The last set $T_{s}$ may have fewer entries. Employing RIP yields then

$$
\begin{aligned}
\left\|\Phi x_{S^{c}}\right\| & \leq \sum_{i=1}^{s}\left\|\Phi x_{T_{i}}\right\| \leq(1+\eta)^{1 / 2} \sum_{i=1}^{s}\left\|x_{T_{i}}\right\| \\
& \leq(1+\eta)^{1 / 2}\left(\sigma_{k}(x)_{\ell_{2}}+\sum_{i=1}^{s-1} k^{-1 / 2}\left\|x_{T_{i}}\right\|_{\ell_{1}}\right) \leq(1+\eta)^{1 / 2}\left(\sigma_{k}(x)_{\ell_{2}}+\frac{\sigma_{k}(x)_{\ell_{1}}}{\sqrt{k}}\right) .
\end{aligned}
$$

If we use this in (3.2) we arrive at (3.3).

\section{A thresholding algorithm without thinning}

The scheme SThresh invokes a thinning step at each iteration. It is not clear whether this is necessary for the successful performance of this algorithm. This prompts us to consider what can be proved without such thinning. In this section, we shall introduce and analyze a greedy algorithm based only on thresholding for the decoding of the information $y=\Phi x+e$. We shall see that we obtain instance optimality in probability except for a small additive factor that can be made as small as we wish (as $n, N \rightarrow \infty$ ).

To this end, we shall need an additional property of the family of random matrices that can be formulated as follows:

P2: For any $z \in \mathbb{R}^{n}, l \in\{1, \ldots, N\}$, and $\delta>0$, there is a set $\Omega_{2}(z, \delta, l)$ such that

$$
\left|\left\langle z, \phi_{l}\right\rangle\right| \leq \delta\|z\|_{\ell_{2}}, \quad \omega \in \Omega_{2}(z, \delta, l)
$$

and

$$
\rho\left(\Omega_{2}^{c}(z, \delta, l)\right) \leq b_{2} e^{-c_{2} n \delta^{2}}
$$

where $b_{2}$ and $c_{2}$ are absolute constants.

Throughout this section we shall assume that for each $n, N$, the $n \times N$ matrices $\Phi(\omega)$ satisfy $\mathbf{P 1}, \mathbf{P} 2$ and that the entries in $\Phi$ are independent identically distributed draws of a single random variable. In particular, the results of this section cover the random Bernouli and Gaussian matrices.

We shall assume throughout this section that the number of measurements factors as $n=a m$ where both $a$ and $m$ are integers. We define $\Phi_{1}$ to be the submatrix of $\Phi$ consisting of its first $m$ rows, $\Phi_{2}$ the submatrix of $\Phi$ consisting of the next $m$ rows and 
so on up to $\Phi_{a}$. Each of these matrices is a randomly drawn $m \times N$ matrix. We will generally denote such a generic $m \times N$ randomly drawn matrix as $\Phi_{0}$.

We now describe a thresholding algorithm for decoding $y=\Phi(x)$. Given a set $\Lambda \subset$ $\{1,2, \ldots, N\}$ of column indices, we denote by $P_{\Lambda}(y)$ the projection of $y$ onto $\operatorname{span}\left\{\phi_{j}\right\}_{j \in \Lambda}$. We also denote by $X(\Lambda)$ the linear space of all $x \in \mathbb{R}^{N}$ which are supported on $\Lambda$. The algorithm will find a set of column indices $\Lambda=\Lambda(y)$ which will be used to decode $y$ as follows: Writing $P_{\Lambda}(y)=\sum_{i \in \Lambda} x_{i}^{\Lambda} \phi_{i}$, and denoting by $\bar{x} \in \mathbb{R}^{N}$ the vector defined by $\bar{x}_{i}:=x_{i}^{\Lambda}, i \in \Lambda, \bar{x}_{i}=0, i \notin \Lambda$, we set

$$
\Delta(y)=\bar{x} .
$$

To find the set $\Lambda$ used in the definition (6.3), we start with $\bar{\Lambda}_{0}=\Lambda_{0}:=\emptyset$. At the $j$-th step of the algorithm, the algorithm will find a set $\Lambda_{j}$ of new coordinates. This is added to the existing "activated" coordinates to give $\bar{\Lambda}_{j}:=\cup_{i=0}^{j} \Lambda_{i}=\cup_{i=1}^{j} \Lambda_{i}$ as the current set of coordinates in our approximation to $x$. We do not want to ever have more than $2 k$ coordinates in our final set $\Lambda(y)$. So we stop the algorithm as soon as $\# \bar{\Lambda}_{j}>2 k$. In fact, we trim the last set of coordinates found in order to be sure the final set $\Lambda(y)$ has cardinality $\leq 2 k$.

Given $i \in\{1, \ldots, a\}$, we denote by $y_{[i]}$ and $e_{[i]}$ the portion of $y, e$, respectively, obtained by setting to zero all coordinates of $y, e$ whose indices are not in $\{(i-1) m+1, \ldots, i m\}$ while keeping the remaining coordinates intact. Suppose $\delta \in(0,1)$ is a given threshold tolerance. At present, we put no restrictions on $\delta$ but later the validity of our theorems will require $\delta$ to be sufficiently small but fixed.

At the first step, we define $r^{1}:=y_{[1]}=\Phi_{1}(x)+e_{[1]}$, compute $\left\|y_{[1]}\right\|$ and consider all coordinates $\nu$ for which

$$
\left|\left\langle r^{1}, \phi_{\nu}^{1}\right\rangle\right| \geq \delta k^{-1 / 2}\left\|r^{1}\right\|
$$

Assume for the moment that there are at most $2 k$ coordinates $\nu$ satisfying (6.4). Then we take $\bar{\Lambda}_{1}:=\Lambda_{1}$ as the set of first activated coordinates and define $X\left(\bar{\Lambda}_{1}\right)$ and compute

$$
x^{1}:=\underset{z \in X\left(\bar{\Lambda}_{1}\right)}{\operatorname{argmin}}\left\|\Phi_{1} z-y_{[1]}\right\|=\left\|y_{[1]}-P_{\bar{\Lambda}_{1}} y_{[1]}\right\| .
$$

The vector $x^{1}$ is the solution to a least squares problem and has a simple closed form representation. The Gramian matrix which needs to be inverted to compute $x^{1}$ is nonsingular with high probability because of the RIP property given below. Finally, we define $r^{2}:=y_{[2]}-\Phi_{2} x^{1}$.

If there are more than $2 k$ coordinates satisfying (6.4) we define $\bar{\Lambda}_{1}:=\Lambda_{1}$ as the set of $2 k$ coordinates which have the largest inner product in (6.4) (with ties handled arbitrarily). We compute $x^{1}$ and $r^{2}$ for this trimmed set as before. We stop the algorithm and output $a^{*}:=1$ and $\Lambda(y):=\Lambda_{1}$ and $\bar{x}:=x^{1}$ as our decoding.

The general step of the algorithm is the following. At the start of the $j$-th step of the algorithm, we have $r^{j}:=y_{[j]}-\Phi_{j} x^{j-1}$. We consider the set of all coordinates $\nu$ such that

$$
\left|\left\langle r^{j}, \phi_{\nu}^{j}\right\rangle\right| \geq \delta k^{-1 / 2}\left\|r^{j}\right\|
$$

If the union of this new set of coordinates together with $\bar{\Lambda}_{j-1}$ has cardinality $\leq 2 k$, we take $\Lambda_{j}$ as the set of all these coordinates and define $\bar{\Lambda}_{j}:=\bar{\Lambda}_{j-1} \cup \Lambda_{j}$ and

$$
x^{j}:=\underset{z \in X\left(\bar{\Lambda}_{j}\right)}{\operatorname{argmin}}\left\|y_{[j]}-\Phi_{j} z\right\|=\left\|y_{[j]}-P_{\bar{\Lambda}_{j}} y_{[j]}\right\| .
$$


If the cardinality of $\bar{\Lambda}_{j-1} \cup \Lambda_{j}$ exceeds $2 k$, we apply trimming. Namely, we define $\Lambda_{j}$ as the subset of coordinates from (6.6) with largest inner products such that the resulting set $\bar{\Lambda}_{j}:=\bar{\Lambda}_{j-1} \cup \Lambda_{j}$ has cardinality $2 k$. In the latter case we stop the algorithm and output $\Lambda(y):=\bar{\Lambda}_{j}, a^{*}:=j$, and $\bar{x}:=x^{j}$.

If the algorithm has not been stopped by a trimming step then we stop it when $j=a$ and output $a^{*}=a, \Lambda(y)=\bar{\Lambda}_{a}$ and $\bar{x}=x^{a}$ as our decoding of $y$. Here, trimming is applied on this last set if necessary to keep $\Lambda(y)$ to have cardinality $\leq 2 k$.

We summarize the scheme as follows:

\section{ThreshII:}

(1) Set $\bar{\Lambda}_{0}=\Lambda_{0}:=\emptyset, x^{0}:=0, j=1, r^{1}=y_{[1]}$;

(2) Define $\Lambda_{j}$ consist of those $\nu$ such that the inner product $\left\langle r^{j}, \phi_{\nu}\right\rangle$ satisfy (6.6).

(3) If $\#\left(\bar{\Lambda}_{j-1} \cup \Lambda_{j}\right)<2 k$, set $\bar{\Lambda}_{j}:=\bar{\Lambda}_{j-1} \cup \Lambda_{j}$, compute $x^{j}$ according to (6.7), set $r^{j+1}=y_{[j+1]}-\Phi_{j+1} x^{j}$ and $j+1 \rightarrow j$ and go to $(2)$.

(4) If $\#\left(\bar{\Lambda}_{j-1} \cup \Lambda_{j}\right) \geq 2 k$ or if $j=a$, define $\bar{\Lambda}_{j}$ by trimming this set, and output $a^{*}=j$ $\bar{x}:=x^{j}$ computed according to $(6.7)$.

Note that each of the quantities appearing above is stochastic and depends on the draw $\omega \in \Omega$, i.e. we have $\Phi_{j}=\Phi_{j}(\omega), x^{j}=x^{j}(\omega)$, but to avoid cluttering of notation we often suppress this dependence in notation when it is clear from the context.

\section{Analysis of algorithm ThreshII}

The main result about ThreshII reads as follows.

Theorem 7.1 Given any $0<\delta \leq \frac{1}{8 \sqrt{3}}$. The thresholding decoder applied with this choice of $\delta$ to $n \times N$ random matrices, $n=$ am, satisfying $\mathbf{P 1}$ and $\mathbf{P 2}$, satisfies the following. For any $x \in \mathbb{R}^{N}$ and any $1 \leq k \leq N$, there exists a set $\Omega_{4}=\Omega_{4}(x, k)$ satisfying

$$
\rho\left(\Omega_{4}^{c}\right) \leq a\left(b_{0} e^{-c_{0} m / 16+3 k \log \left(\frac{24 N}{3 k}\right)}+b_{1} e^{-c_{1} m / 4}+\left(2 b_{1}+b_{2}\right) N e^{-c m \delta^{2} / k}+b_{1} e^{-c_{1} m \delta^{2}}\right),
$$

such that for any $\omega$ in $\Omega_{4}$ and any noise vector $e$, the decoded vector $\bar{x}$ of the above greedy decoder satisfies

$$
\|x-\bar{x}\|_{\ell_{2}} \leq 2^{-a / 2}\|x\|+C^{*}\left(\sigma_{k}(x)+\max _{j=1, \cdots, a^{*}}\left\|e_{[j]}\right\|\right)
$$

where $C^{*}:=\max \left\{\sqrt{408},\left[1+3 \sqrt{3}+\frac{4 \sqrt{3}}{\delta}\right]\right\}$.

We have the following corollary to this theorem. 
Corollary 7.2 Suppose that $r, s>0$ are given and that the random process generates $m \times N$ matrices $\Phi_{0}(\omega)$ which satisfy $\mathbf{P 1}$ and $\mathbf{P 2}$. We use $n \times N$ matrices $\Phi(\omega), \omega \in \Omega$, with $n=$ am and $a:=\lceil 2 r \log N\rceil$, for encoding and use the thresholding algorithm with $\delta \in\left(0, \frac{1}{8 \sqrt{3}}\right]$ for decoding. Then, for a sufficiently small constant $c(\delta)>0$ we have the following. For each $x \in \mathbb{R}^{N}$, there is a set $\Omega_{4} \subset \Omega$ with

$$
\rho\left(\Omega_{4}^{c}\right) \leq N^{-s}
$$

such that for any draw $\omega \in \Omega_{4}$ and and any noise vector e, one has for each $k \leq$ $c(\delta, r) n /(\log N)^{2}$

$$
\|x-\bar{x}\| \leq N^{-r}+C\left(\sigma_{k}(x)+\max _{j=1, \cdots, a^{*}}\left\|e_{[j]}\right\|\right),
$$

with $C=C(\delta)$ depending only on $\delta$.

Proof: We apply Theorem 7.1 with the values of $a$ and $\delta$ as specified in the statement of the Corollary. We can take $\Omega_{4}$ as the set in that theorem. Then $\rho\left(\Omega_{4}^{c}\right)$ is bounded by (7.1). The second and fourth terms on the right hand side of (7.1) are both less or equal to $C^{\prime} a e^{-c^{\prime} m}$ and so is the first term if $c(\delta, r)$ is small enough, for the range of $k$ described in the theorem. The remaining third term is bounded by $a N\left(2 b_{1}+b_{2}\right) e^{-\frac{c \log N}{c(\delta, r)}}$. Thus each of these terms can be bounded by $N^{-s} / 4$ provided $c(\delta, r)$ is small enough and we therefore obtain (7.3). The estimate (7.4) follows from (7.2) because $2^{-a / 2} \leq N^{-r}$.

The remainder of this section is devoted to the proof of Theorem 7.1 and a short discussion of its ramifications. The proof is somewhat simpler when the noise $e$ in the observation is zero and the reader may wish to make that assumption on first reading. Throughout the remainder of this section, for a given but fixed $x \in \mathbb{R}^{N}$ and a given $k$, we let $S_{k}$ denote a set of its largest $k$ coordinates.

In accordance with the above initialization we shall define $x^{0}:=0$ for the purposes of the analysis that follows below. We begin with the following lemma which bounds $\left\|x-x^{j}\right\|$ by a multiple of $\left\|y_{[j]}-\Phi_{j} x^{j}\right\|$. Note that $x^{j}$ is stochastically dependent on $\Phi_{j}$.

Lemma 7.3 Given $x \in \mathbb{R}^{N}$ and $k \geq 1$, define

$$
\Omega_{3}:=\Omega_{3}(x, k):=\cap_{j=1}^{a}\left[\Omega_{0}\left(3 k, 1 / 2, \Phi_{j}\right) \cap \Omega_{1}\left(x_{S_{k}^{c}}, 1 / 2, \Phi_{j}\right)\right]
$$

where the sets $\Omega_{0}$ correspond to $\mathbf{R I P}$ and the sets $\Omega_{1}$ correspond to $\mathbf{P} \mathbf{1}$. Then,

$$
\rho\left(\Omega_{3}^{c}\right) \leq b_{0} a e^{-c_{0} m / 16+3 k \log \left(\frac{24 N}{3 k}\right)}+b_{1} a e^{-c_{1} m / 4},
$$

and for each $\omega \in \Omega_{3}$ and $1 \leq j \leq a^{*}$, we have $\left(\right.$ for $\left.x^{j}=x^{j}(\omega)\right)$

$$
\left\|x-x^{j}\right\| \leq(1+\sqrt{3}) \sigma_{k}(x)+\sqrt{2}\left(\left\|y_{[j]}-\Phi_{j} x^{j}\right\|+\left\|e_{[j]}\right\|\right) .
$$

Proof: We first check the measure of $\Omega_{3}^{c}$. According to Properties P1 and RIP (see (2.4)) we have

$$
\rho\left(\Omega_{3}^{c}\right) \leq \sum_{j=1}^{a} \rho\left(\Omega_{0}\left(3 k, 1 / 2, \Phi_{j}\right)^{c}\right)+\sum_{j=1}^{a} \rho\left(\Omega_{1}\left(x_{S_{k}^{c}}, 1 / 2, \Omega_{j}\right)^{c}\right)
$$




$$
\leq b_{0} a e^{-c_{0} m / 16+3 k \log \left(\frac{24 N}{3 k}\right)}+b_{1} a e^{-c_{1} m / 4}
$$

This proves $(7.6)$

To verify (7.7), we have

$$
\left\|x-x^{j}\right\| \leq\left\|x-x_{S_{k}}\right\|+\left\|x_{S_{k}}-x^{j}\right\|=\sigma_{k}(x)+\left\|x_{S_{k}}-x^{j}\right\| .
$$

We know that $x_{S_{k}}-x^{j}$ is $3 k$-sparse if $j \leq a^{*}$. Hence, for $\omega \in \Omega_{3}$, we have from $\operatorname{RIP}(3 k, 1 / 2)$,

$$
\left\|x_{S_{k}}-x^{j}\right\| \leq \sqrt{2}\left\|\Phi_{j} x_{S_{k}}-\Phi_{j} x^{j}\right\|, \quad 1 \leq j<a^{*} .
$$

This gives for $1 \leq j \leq a^{*}$,

$$
\left\|x_{S_{k}}-x^{j}\right\| \leq \sqrt{2}\left\{\left\|\Phi_{j} x_{S_{k}}-\Phi_{j} x\right\|+\left\|\Phi_{j}\left(x-x^{j}\right)\right\|\right\}=\sqrt{2}\left\{\left\|\Phi_{j} x_{S_{k}^{c}}\right\|+\left\|\Phi_{j}\left(x-x^{j}\right)\right\|\right\} .
$$

Since, by $\mathbf{P} 1,\left\|\Phi_{j} x_{S_{k}^{c}}\right\| \leq \sqrt{3 / 2}\left\|x_{S_{k}^{c}}\right\|=\sqrt{3 / 2} \sigma_{k}(x)$ and since

$$
y_{[j]}-\Phi_{j} x^{j}=\Phi_{j}\left(x-x^{j}\right)+e_{[j]},
$$

we have proved (7.7).

Our next two lemmas are going to show the quantitative effects of thresholding and will later be used to provide error bounds for our algorithm.

Lemma 7.4 Let $\delta \in(0,1), u \in \mathbb{R}^{N}$, and let $\Lambda:=\Lambda(u, \delta, k)$ be the set of all indices $\nu$ such that $\left|u_{\nu}\right| \geq \delta k^{-1 / 2}\|u\|$. Then,

$$
\left\|u-u_{\Lambda}\right\|^{2}=\sum_{\nu \notin \Lambda}\left|u_{\nu}\right|^{2} \leq 3 \delta^{2}\|u\|^{2}+\sigma_{3 k}^{2}(u) .
$$

Proof: Let $\Lambda_{0}$ be a set of the $3 k$ largest coordinates of $u$ so that $\sum_{\nu \notin \Lambda_{0}}\left|u_{\nu}\right|^{2}=\sigma_{3 k}^{2}(u)$. We have

$$
\sum_{\nu \notin \Lambda}\left|u_{j}\right|^{2} \leq \sum_{\nu \in \Lambda_{0} \cap \Lambda^{c}}\left|u_{\nu}\right|^{2}+\sum_{\nu \in \Lambda_{0}^{c} \cap \Lambda^{c}}\left|u_{\nu}\right|^{2} \leq 3 k \delta^{2}\|u\|^{2} / k+\sigma_{3 k}^{2}(u)=3 \delta^{2}\|u\|^{2}+\sigma_{3 k}^{2}(u)
$$

where we used the fact that $\Lambda_{0}$ has cardinality $3 k$.

Lemma 7.5 Let $u \in \mathbb{R}^{N}$ and let $v:=\Phi_{0}(u)+e_{[0]}, e_{[0]} \in \mathbb{R}^{n}$, where $\Phi_{0}=\Phi_{0}(\omega)$ is an $m \times N$ matrix randomly drawn from our stochastic process which satisfies $\mathbf{P} 1$ and $\mathbf{P} 2$. Moreover, assume that

$$
\left\|e_{[0]}\right\| \leq\|u\| / 4
$$

Let $\Lambda^{\prime}(v, \delta, k, \omega)$ be the set of all $\nu$ such that

$$
\left|\left\langle v, \phi_{\nu}\right\rangle\right| \geq \delta\|v\| k^{-1 / 2}
$$

Then, there is a set $\Omega\left(u, \delta, k, \Phi_{0}\right)$ such that

$$
\rho\left(\Omega^{c}\left(u, \delta, k, \Phi_{0}\right)\right) \leq\left(2 b_{1}+b_{2}+1\right) N e^{-\frac{c m \delta^{2}}{k}}
$$


where $c:=\min \left(c_{1}, c_{2}\right) / 64$ and $b_{1}, b_{2}, c_{1}, c_{2}$ are the constants in $\mathbf{P} \mathbf{1}$ and $\mathbf{P} \mathbf{2}$, and for any $\omega \in \Omega\left(u, \delta, k, \Phi_{0}\right)$ with $\delta \leq 1 / 12$, we have

$$
\Lambda(u, 2 \delta, k) \subset \Lambda^{\prime}(v, \delta, k, \omega)
$$

and

$$
\Lambda^{\prime}(v, \delta, k, \omega) \subset \Lambda(u, \delta / 2, k)
$$

where the set $\Lambda(u, \delta, k)$ is defined in Lemma $\% .4$.

Proof: For each $\nu \in \Lambda(u, \delta, k)$, let $u(\nu):=u-u_{\nu} \delta_{\nu}$ and

$$
v(\nu):=\Phi_{0}(u(\nu))+e_{[0]}=v-u_{\nu} \phi_{\nu}
$$

where $\delta_{\nu} \in \mathbb{R}^{N}$ is the $\nu$ th coordinate vector. It follows that $\|u(\nu)\| \leq\|u\|$ for each $\nu$ and $v=u_{\nu} \phi_{\nu}+v(\nu)$. According to Property $\mathbf{P} \mathbf{1}$, for each $\nu$, there is a set $\Omega_{1}\left(u(\nu), \delta, \Phi_{0}\right)$ such that

$$
\rho\left(\Omega_{1}\left(u(\nu), \delta, \Phi_{0}\right)^{c}\right) \leq b_{1} e^{-c_{1} m \delta^{2}}
$$

and for all $\omega \in \Omega_{1}\left(u(\nu), \delta, \Phi_{0}\right)$,

$$
\|v(\nu)\| \leq \sqrt{1+\delta}\|u(\nu)\|+\left\|e_{[0]}\right\| \leq(\sqrt{1+\delta}+1 / 4)\|u\|, \quad \omega \in \Omega_{1}\left(u(\nu), \delta, \Phi_{0}\right),
$$

where we have used the assumption that $\left\|e_{[0]}\right\| \leq\|u\| / 4$. Observe that $v(\nu)$ is stochastically independent of $\phi_{\nu}$. Therefore, according to Property $\mathbf{P} 2$, there is a set $\Omega_{2}\left(v(\nu), \delta /(8 \sqrt{k}), \nu, \Phi_{0}\right)$ with

$$
\rho\left(\Omega_{2}\left(v(\nu), \delta /(8 \sqrt{k}), \nu ; \Phi_{0}\right)^{c}\right) \leq b_{2} e^{-\frac{c_{2} m \delta^{2}}{64 k}}
$$

and, such that for all $\omega \in \Omega_{2}\left(v(\nu), \delta /(8 \sqrt{k}), \nu, \Phi_{0}\right)$, we have

$$
\begin{aligned}
\left|\left\langle v(\nu), \phi_{\nu}\right\rangle\right| & \leq \frac{\delta\|v(\nu)\|}{8 \sqrt{k}} \leq \frac{\delta(\sqrt{1+\delta}+1 / 4)\|u(\nu)\|}{8 \sqrt{k}} \\
& \leq \frac{\delta \sqrt{1+\delta}\|u\|}{6 \sqrt{k}}, \quad \omega \in \Omega_{2}\left(u(\nu), \delta, \nu ; \Phi_{0}\right)
\end{aligned}
$$

where we have used (7.20) and the fact that $\|u(\nu)\| \leq\|u\|$.

We now define

$$
\begin{aligned}
& \Omega\left(u, \delta, k, \Phi_{0}\right):= \\
& \bigcap_{\nu \in \Lambda(u, \delta, k)}\left(\Omega_{1}\left(u(\nu), \delta, \Phi_{0}\right) \cap \Omega_{1}\left(\delta_{\nu}, \delta, \Phi_{0}\right) \cap \Omega_{2}\left(v(\nu), \delta /(8 \sqrt{k}), \nu, \Phi_{0}\right)\right) \cap \Omega_{1}\left(u, \delta, \Phi_{0}\right),
\end{aligned}
$$

Then, this set satisfies (7.16) because of (7.19), (7.21) and property $\mathbf{P} \mathbf{1}$ applied to $\delta_{\nu}$ and $u$.

We now prove (7.17). For any $\omega \in \Omega\left(u, \delta, k, \Phi_{0}\right)$ and any $\nu \in \Lambda(u, 2 \delta, k)$, by $(7.22)$,

$$
\begin{aligned}
\left|\left\langle v, \phi_{\nu}\right\rangle\right| & \geq\left|u_{\nu}\right|(1-\delta)-\left|\left\langle v(\nu), \phi_{\nu}\right\rangle\right| \\
& \geq 2 k^{-1 / 2} \delta(1-\delta)\|u\|-k^{-1 / 2}(\delta / 6) \sqrt{1+\delta}\|u\| .
\end{aligned}
$$


Invoking $\mathbf{P} 1$ with respect to $u$, we conclude that

$$
\begin{aligned}
\left|\left\langle v, \phi_{\nu}\right\rangle\right| & \geq \frac{\delta}{\sqrt{k}}\left(2(1-\delta)-\frac{\sqrt{1+\delta}}{6}\right) \frac{\left\|\Phi_{0} u\right\|}{\sqrt{1+\delta}} \\
& \geq \frac{\delta}{\sqrt{k}}\left(2(1-\delta)-\frac{\sqrt{1+\delta}}{6}\right) \frac{1}{\sqrt{1+\delta}}\left(\|v\|-\left\|e_{[0]}\right\|\right) .
\end{aligned}
$$

Now observe, again by $\mathbf{P} \mathbf{1}$, that (7.14) implies

$$
\left\|e_{[0]}\right\| \leq \frac{1}{4 \sqrt{1+\delta}}\left\|\Phi_{0} u\right\| \leq \frac{1}{4 \sqrt{1+\delta}}\left(\|v\|+\left\|e_{[0]}\right\|\right),
$$

whence one infers

$$
\left\|e_{[0]}\right\| \leq \frac{1}{4(\sqrt{1+\delta}-1 / 4)}\|v\| \leq \frac{1}{3}\|v\| .
$$

Thus, combining (7.24) and (7.25), gives

$$
\left|\left\langle v, \phi_{\nu}\right\rangle\right| \geq\left\{\frac{2}{3 \sqrt{1+\delta}}\left(2(1-\delta)-\frac{\sqrt{1+\delta}}{6}\right)\right\} \frac{\delta}{\sqrt{k}}\|v\| .
$$

One can verify that for $\delta \leq 1 / 10$ the factor in curly brackets is indeed larger than 1 which shows (7.17).

We now prove (7.18). For any $\omega \in \Omega\left(u, \delta, k, \Phi_{0}\right)$ and any $\nu \notin \Lambda(u, \delta / 2, k)$, we use again (7.22) and $\mathbf{P} \mathbf{1}$ for $u$ to conclude that

$$
\begin{aligned}
\left|\left\langle v, \phi_{\nu}\right\rangle\right| & \leq\left|u_{\nu}\right|(1+\delta)+\left|\left\langle v(\nu), \phi_{\nu}\right\rangle\right| \\
& \leq(1+\delta)(\delta / 2) k^{-1 / 2}\|u\|+k^{-1 / 2}(\delta / 6) \sqrt{1+\delta}\|u\| \\
& \leq \frac{\delta}{\sqrt{k}}\left(\frac{1+\delta}{2}+\frac{\sqrt{1+\delta}}{6}\right) \frac{1}{\sqrt{1-\delta}}\left\|\Phi_{0} u\right\| \\
& \leq \frac{\delta}{\sqrt{k}}\left(\frac{1+\delta}{2}+\frac{\sqrt{1+\delta}}{6}\right) \frac{1}{\sqrt{1-\delta}}\left(\|v\|+\left\|e_{[0]}\right\|\right) \\
& \leq \frac{\delta}{\sqrt{k}}\left(\frac{1+\delta}{2}+\frac{\sqrt{1+\delta}}{6}\right) \frac{4}{3 \sqrt{1-\delta}}\|v\|,
\end{aligned}
$$

where we have used (7.25) in the last step. One can verify that for $\delta \leq 1 / 12$ one has $\left(\frac{1+\delta}{2}+\frac{\sqrt{1+\delta}}{6}\right) \frac{4}{3 \sqrt{1-\delta}}<1$ so that $\left|\left\langle v, \phi_{\nu}\right\rangle\right|<\delta k^{-1 / 2}\|u\|$. This shows that any such $\nu$ could not have been chosen for $\Lambda^{\prime}(v, \delta, k, \omega)$. This completes the proof of the lemma.

We can now define the set $\Omega_{4}$ that appears in the statement of Theorem 7.1 and Corollary 7.2 as

$$
\Omega_{4}:=\Omega_{4}(x, k, \delta):=\Omega_{3}(x, k) \cap \cap_{j=1}^{a^{*}}\left[\Omega\left(x-x^{j-1}, \delta, k, \Phi_{j}\right) \cap \Omega_{1}\left(x_{S_{k}^{c}}, \delta, \Phi_{j}\right)\right],
$$

where $\Omega_{3}$ is the set in Lemma 7.3, the next sets in brackets come from Lemma 7.5 and the last sets come from $\mathbf{P} \mathbf{1}$. Let us note that

$$
\rho\left(\Omega_{4}(x, k, \delta)^{c}\right) \leq a \quad\left\{\left(b_{0} e^{-c_{0} m / 16+3 k \log \left(\frac{24 N}{3 k}\right)}+b_{1} e^{-c_{1} m / 4}\right.\right.
$$




$$
\left.+\left(2 b_{1}+b_{2}+1\right) N e^{-c m \delta^{2} / k}+b_{1} e^{-c_{1} m \delta^{2}}\right\}
$$

Indeed, this estimate follows from (7.6), (7.16) and P1 and the fact that $a^{*} \leq a$. The set $\Omega_{4}$ will be used in the remainder of this section.

The next lemma shows a certain reduction of the error at each iteration of the algorithm.

Lemma 7.6 For each $1 / 12 \geq \delta>0$, each $x \in \mathbb{R}^{N}$, each $\omega \in \Omega_{4}(x, k, \delta)$ and each $1 \leq j<a^{*}$, we have

$$
\left\|x-x^{j}\right\|^{2} \leq A\left\|x-x^{j-1}\right\|^{2}+B \sigma_{k}^{2}(x)+C\left\|e_{[j]}\right\|^{2},
$$

where $A:=96 \delta^{2}, B:=204$, and $C=196$. This same estimate holds for $j=a^{*}$ provided this last set was not trimmed.

Proof: We fix a value of $j$ and assume that $\omega \in \Omega_{4}(x, k, \delta)$. At the beginning of the $j$-th step of the decoding we have in hand $\bar{\Lambda}_{j-1}$ and $x^{j-1}$ where, according to our initialization of the algorithm, $x^{0}:=0$ and $\bar{\Lambda}_{0}:=\emptyset$. Thresholding on the vector $\Phi_{j}^{*} r^{j}=\Phi_{j}^{*}\left(\Phi_{j}(x-\right.$ $\left.\left.x^{j-1}\right)+e_{[j]}\right)$, now gives the set $\Lambda_{j}$ and the new composite set $\bar{\Lambda}_{j}$. By our assumption on $j$, there was no trimming involved.

We shall distinguish between two cases: (a) $\left\|e_{[j]}\right\| \leq\left\|x-x^{j-1}\right\| / 4$ and (b) $\left\|e_{[j]}\right\|>$ $\left\|x-x^{j-1}\right\| / 4$.

In case (a), since $\Phi_{j}$ is drawn independently of $x-x^{j-1}$, we can apply Lemma 7.5 for $u:=x-x^{j-1}$ and $e_{[0]}:=e_{[j]}$. It says that for $\omega \in \Omega\left(x-x^{j-1}, \delta, k, \Phi_{j}\right)$, the set $\Lambda_{j}$ contains all coordinates $\nu$ for which $\left|x_{\nu}-x_{\nu}^{j-1}\right| \geq 2 \delta k^{-1 / 2}\left\|x-x^{j-1}\right\|$. Hence, we can apply Lemma 7.4 to $u=x-x^{j-1}$ and obtain for $w:=x^{j-1}+\left(x-x^{j-1}\right)_{\Lambda_{j}}$, upon noting that $\left(x-x^{j-1}\right)_{\Lambda_{j}^{c}}=x-w$,

$$
\|x-w\|^{2} \leq 12 \delta^{2}\left\|x-x^{j-1}\right\|^{2}+\sigma_{3 k}^{2}\left(x-x^{j-1}\right) \leq 12 \delta^{2}\left\|x-x^{j-1}\right\|^{2}+\sigma_{k}^{2}(x),
$$

where the last inequality uses the fact that $\sigma_{3 k}\left(x-x^{j-1}\right) \leq \sigma_{k}(x)$ because $x^{j-1}$ is in $\Sigma_{2 k}$.

Starting with Lemma 7.3, we can now estimate

$$
\begin{aligned}
\left\|x-x^{j}\right\| & \leq(1+\sqrt{3}) \sigma_{k}(x)+\sqrt{2}\left(\left\|y_{[j]}-\Phi_{j} x^{j}\right\|+\left\|e_{[j]}\right\|\right) \\
& \leq(1+\sqrt{3}) \sigma_{k}(x)+\sqrt{2}\left(\left\|y_{[j]}-\Phi_{j} w\right\|+\left\|e_{[j]}\right\|\right) \\
& \leq(1+\sqrt{3}) \sigma_{k}(x)+\sqrt{2}\left(\left\|\Phi_{j}(x-w)\right\|+2\left\|e_{[j]}\right\|\right)
\end{aligned}
$$

where the second to last inequality uses the minimality of the least squares solution in the space $X\left(\bar{\Lambda}_{j}\right)$ of vectors in $\mathbb{R}^{N}$ supported in $\bar{\Lambda}_{j}$.

We now want to estimate the middle term in (7.32). We cannot use $\mathbf{P} \mathbf{1}$ directly because $w$ depends on $\Phi_{j}$. Instead, we write $x-w=x-x_{S_{k}}+\left(x_{S_{k}}-w\right)$ and find

$$
\begin{aligned}
\left\|\Phi_{j}(x-w)\right\| & \leq\left\|\Phi_{j}\left(x-x_{S_{k}}\right)\right\|+\left\|\Phi_{j}\left(x_{S_{k}}-w\right)\right\| \\
& \leq \sqrt{1+\delta}\left(\left\|x_{S_{k}}\right\|+\left\|x_{S_{k}}-w\right\|\right) \\
& \leq \sqrt{1+\delta}\left(\sigma_{k}(x)+\left\|x-x_{S_{k}}\right\|+\|x-w\|\right) \\
& \leq \sqrt{1+\delta}\left(2 \sigma_{k}(x)+\|x-w\|\right) .
\end{aligned}
$$


Here in the second inequality we used $\mathbf{P} \mathbf{1}$ for $x_{S_{k}^{c}}$ and $\mathbf{R I P}(3 k, \delta)$ for $\Phi_{j}$.

We now subsitute (7.33) into (7.32) to obtain

$$
\begin{aligned}
\left\|x-x^{j}\right\| & \leq(1+\sqrt{3}+2 \sqrt{2} \sqrt{1+\delta}) \sigma_{k}(x)+\sqrt{2} \sqrt{1+\delta}\|x-w\|+2 \sqrt{2}\left\|e_{[j]}\right\| \\
& \leq 7 \sigma_{k}(x)+2\|x-w\|+2 \sqrt{2}\left\|e_{[j]}\right\|,
\end{aligned}
$$

because $0<\delta<1$. We square this last inequality and then use (7.31) to arrive at

$$
\begin{aligned}
\left\|x-x^{j}\right\|^{2} & \leq 2\left(7 \sigma_{k}^{2}(x)+2 \sqrt{2}\left\|e_{[j]}\right\|\right)^{2}+8\|x-w\|^{2} \\
& \leq 204 \sigma_{k}^{2}(x)+96 \delta^{2}\left\|x-x^{j-1}\right\|^{2}+32\left\|e_{[j]}\right\|^{2} \\
& \leq A\left\|x-x^{j-1}\right\|^{2}+B \sigma_{k}^{2}(x)+C\left\|e_{[j]}\right\|^{2},
\end{aligned}
$$

as desired.

Now we turn to case (b) $\left\|e_{[j]}\right\|>\left\|x-x^{j-1}\right\| / 4$, and use again that $\Phi_{j}$ is drawn independently of $x-x^{j-1}$, so that $\mathbf{P} 1$ yields

$$
\begin{aligned}
\left\|e_{[j]}\right\| & \geq \frac{1}{4 \sqrt{1+\delta}}\left\|\Phi_{j}\left(x-x^{j-1}\right)\right\| \\
& =\frac{1}{4 \sqrt{1+\delta}}\left\|y_{[j]}-\Phi_{j} x^{j-1}-e_{[j]}\right\| \\
& \geq \frac{1}{4 \sqrt{1+\delta}}\left(\left\|y_{[j]}-\Phi_{j} x^{j-1}\right\|-\left\|e_{[j]}\right\|\right) .
\end{aligned}
$$

Since $x^{j}$ minimizes $\left\|y_{[j]}-\Phi_{j} z\right\|$ over $X\left(\bar{\Lambda}_{j}\right)$, and $\bar{\Lambda}_{j}$ contains the support of $x^{j-1}$, we conclude that

$$
(4 \sqrt{1+\delta}+1)\left\|e_{[j]}\right\| \geq\left\|y_{[j]}-\Phi_{j} x^{j-1}\right\| \geq\left\|y_{[j]}-\Phi_{j} x^{j}\right\|
$$

Note that $(4 \sqrt{1+\delta}+1) \leq 6$ for $\delta \leq 1 / 10$. We invoke now Lemma 7.3 to conclude that

$$
\left\|x-x^{j}\right\| \leq(1+\sqrt{3}) \sigma_{k}(x)+7 \sqrt{2}\left\|e_{[j]}\right\| .
$$

Squaring both sides confirms (7.30) and finishes the proof.

Proof Theorem 7.1: We can now prove our main result about the greedy algorithm of this section. Let $\delta \leq 1 / 8 \sqrt{3}$. Then $A=96 \delta^{2} \leq 1 / 2$ and $\delta \leq 1 / 12$.

We will continue to denote by $S_{k}$ a set of $k$ coordinates corresponding to the largest entries (in absolute value) of $x$. We shall consider two cases.

Case 1: In this first case, we assume that the algorithm never employed trimming. In particular this means that $a^{*}=a$ and $\bar{x}=x^{a}$. We introduce the abbreviated notation $E_{j}:=\left\|x-x^{j}\right\|^{2}, \eta_{j}:=\left\|e_{[j]}\right\|^{2}$, and $\sigma:=\sigma_{k}^{2}(x)$. Then, an application of Lemma 7.6 at each iteration gives

$$
E_{j} \leq A E_{j-1}+B \sigma+C \eta_{j} \quad j=2, \ldots, a^{*} .
$$

Iteratively applying this inequality gives

$$
\begin{aligned}
\|x-\bar{x}\|^{2} & =\left\|x-x^{a}\right\|^{2} \leq A^{a}\|x\|^{2}+\frac{B}{1-A} \sigma_{k}^{2}(x)+C \sum_{i=0}^{a-1} A^{i} \eta_{j-i} \\
& \leq 2^{-a}\|x\|^{2}+2 B \sigma_{k}^{2}(x)+C\left[\sum_{i=0}^{a-1} A^{i}\right] \max _{j=1, \cdots, a^{*}} \eta_{j}
\end{aligned}
$$


Since $A \leq 1 / 2$, this proves $(7.2)$ in this case.

Case 2: The remaining case we have to consider is when trimming was used to create the last set $\bar{\Lambda}_{a^{*}}$. In this case the following observation is useful.

Remark 7.7 For any $\nu \in \bar{\Lambda}_{a^{*}}$ one has for any $\omega \in \Omega_{4}$

$$
\frac{\delta}{2 \sqrt{k}} \max _{j=1, \cdots, a^{*}}\left\|e_{[j]}\right\|+\left|x_{\nu}\right| \geq \frac{\delta}{2 \sqrt{2 k}}\left\|x-x_{\bar{\Lambda}_{a^{*}}}\right\| .
$$

To prove (7.39), for any $\nu \in \bar{\Lambda}_{a^{*}}$ consider the first iteration $j$ when $\nu \in \Lambda_{j}$. The inner product of $r^{j}$ with $\phi_{\nu}^{j}$ was by definition larger than $\delta k^{-1 / 2}\left\|r^{j}\right\|$. It follows from (7.18) that for $\omega \in \Omega_{4}(x, k, \delta)$

$$
\left|x_{\nu}\right| \geq \frac{\left\|r^{j}\right\| \delta}{2 \sqrt{k}}
$$

Since $x^{j-1}$ is supported on $\bar{\Lambda}_{j-1}$, one obtains

$$
\left\|x-x_{\bar{\Lambda}_{j-1}}\right\| \leq\left\|x-x^{j-1}\right\| \leq(1-\delta)^{-1 / 2}\left\|\Phi_{j}\left(x-x^{j-1}\right)\right\| \leq \sqrt{2}\left(\left\|r^{j}\right\|+\left\|e_{[j]}\right\|\right)
$$

where the last inequality uses that $\omega \in \Omega_{4}$ allows us to apply $\mathbf{P} \mathbf{1}$ for $x-x^{j-1}$ as well as the fact that $\delta \leq 1 / 2$. This confirms (7.39).

Since trimming was used we have $\#\left(\bar{\Lambda}_{a^{*}}\right)=2 k$. It follows that $\bar{\Lambda}_{a^{*}}$ contains at least $k$ coordinates from $S_{k}^{c}$. Since (7.39) holds for each $\nu \in \bar{\Lambda}_{a^{*}} \cap S_{k}^{c}$, it follows that

$$
\sum_{\nu \in \bar{\Lambda}_{a^{*} \cap S_{k}^{c}}}\left(\left|x_{\nu}\right|+\frac{\delta}{2 \sqrt{k}} \max _{j=1, \cdots, a^{*}}\left\|e_{[j]}\right\|\right)^{2} \geq \frac{\delta^{2}}{8}\left\|x-x_{\bar{\Lambda}_{a^{*}}}\right\|^{2} .
$$

On the other hand, we have

$$
\begin{aligned}
\sum_{\nu \in \bar{\Lambda}_{a^{*} \cap S_{k}^{c}}}\left(\left|x_{\nu}\right|+\frac{\delta}{2 \sqrt{k}} \max _{j=1, \cdots, a^{*}}\left\|e_{[j]}\right\|\right)^{2} \leq & 2 \sum_{\nu \in \bar{\Lambda}_{a^{*} \cap S_{k}^{c}}}\left|x_{\nu}\right|^{2} \\
& +2 \frac{\delta^{2} \#\left(\bar{\Lambda}_{\left.a^{*} \cap S_{k}^{c}\right)}\right.}{4 k} \max _{j=1, \cdots, a^{*}}\left\|e_{[j]}\right\|^{2} \\
\leq & 2 \sigma_{k}(x)^{2}+\delta^{2} \max _{j=1, \cdots, a^{*}}\left\|e_{[j]}\right\|^{2} .
\end{aligned}
$$

Thus we conclude that

$$
\left\|x-x_{\bar{\Lambda}_{a^{*}}}\right\| \leq \frac{4}{\delta} \sigma_{k}(x)+2 \sqrt{2} \max _{j=1, \cdots, a^{*}}\left\|e_{[j]}\right\|
$$

where we have used the fact that $\#\left(\bar{\Lambda}_{a^{*}} \cap S_{k}^{c}\right) \geq k$.

We now turn to estimating $\|x-\bar{x}\|$. We begin with

$$
\|x-\bar{x}\|=\left\|x-x^{a^{*}}\right\| \leq\left\|x-x_{S_{k}}\right\|+\left\|x_{S_{k}}-x^{a^{*}}\right\| \leq \sigma_{k}(x)+\left\|x_{S_{k}}-x^{a^{*}}\right\|
$$

The second term was estimated in (7.11) in the proof of Lemma 7.3 (with $j=a^{*}$ ). Using that estimate and the minimality of the least squares solution, we obtain $\|x-\bar{x}\| \leq(1+\sqrt{3}) \sigma_{k}(x)+\sqrt{2}\left\|\Phi_{a^{*}}\left(x-x^{a^{*}}\right)\right\|$ 


$$
\begin{aligned}
& \leq(1+\sqrt{3}) \sigma_{k}(x)+\sqrt{2}\left(\left\|y_{\left[a^{*}\right]}-\Phi_{a^{*}} x^{a^{*}}\right\|+\left\|e_{\left[a^{*}\right]}\right\|\right) \\
& \leq(1+\sqrt{3}) \sigma_{k}(x)+\sqrt{2}\left(\left\|y_{\left[a^{*}\right]}-\Phi_{a^{*}} x_{\bar{\Lambda}_{a^{*}}}\right\|+\max _{j=1, \cdots, a^{*}}\left\|e_{[j]}\right\|\right) \\
& \leq(1+\sqrt{3}) \sigma_{k}(x)+\sqrt{2}\left(\left\|\Phi_{a^{*}}\left(x-x_{S_{k}}\right)\right\|+\left\|\Phi_{a^{*}}\left(x_{S_{k}}-x_{\bar{\Lambda}_{a^{*}}}\right)\right\|+2 \max _{j=1, \cdots, a^{*}}\left\|e_{[j]}\right\| .\right) .
\end{aligned}
$$

Now $\omega \in \Omega_{4} \subset \Omega_{3}$. Looking at the definition of $\Omega_{3}$ in (7.5), we see that we can apply $\mathbf{P} 1$ and RIP to conclude that

$$
\begin{aligned}
\|x-\bar{x}\| & \leq(1+\sqrt{3}) \sigma_{k}(x)+\sqrt{3}\left(\left\|x_{S_{k}^{c}}\right\|+\left\|x_{S_{k}}-x_{\bar{\Lambda}_{a^{*}}}\right\|\right)+2 \sqrt{2} \max _{j=1, \cdots, a^{*}}\left\|e_{[j]}\right\| \\
& \leq(1+2 \sqrt{3}) \sigma_{k}(x)+\sqrt{3}\left(\left\|x-x_{S_{k}}\right\|+\left\|x-x_{\bar{\Lambda}_{a^{*}}}\right\|\right)+2 \sqrt{2} \max _{j=1, \cdots, a^{*}}\left\|e_{[j]}\right\| . \\
& \leq(1+3 \sqrt{3}) \sigma_{k}(x)+\sqrt{3}\left\|x-x_{\bar{\Lambda}_{a^{*}}}\right\|+2 \sqrt{2} \max _{j=1, \cdots, a^{*}}\left\|e_{[j]}\right\| \\
& \leq\left[1+3 \sqrt{3}+\frac{4 \sqrt{3}}{\delta}\right] \sigma_{k}(x)+2(\sqrt{2}+\sqrt{6}) \max _{j=1, \cdots, a^{*}}\left\|e_{[j]}\right\|
\end{aligned}
$$

where the last inequality uses (7.42). This shows that (7.2) holds in the second case as well and completes the proof of the theorem.

We conclude this section with some remarks. The above argument shows that as soon as trimming is necessary, i.e. the sets $\bar{\Lambda}_{j}$ build up fast enough, the decoder is actually instance-optimal in the original sense when $e=0$, see (7.44).

Remark 7.8 Even when trimming does not occur in the algorithm, we still have the following estimate: suppose that $e=0$ and $2 q:=\# \bar{\Lambda}_{a} \leq 2 k$, then one has

$$
\|x-\bar{x}\| \leq(5+\sqrt{3}) \sigma_{k}(x)+\delta^{-1} \sqrt{\frac{32 k}{q}} \sigma_{q}(x) .
$$

Thus, as long as the size of $\bar{\Lambda}_{a^{*}}$, i.e. the support of the decoder output is comparable to $k$, one can bound the error by a constant multiple of the corresponding q-term approximation error.

Proof: Remark 7.7 together with the argument leading to (7.42) yields

$$
\sigma_{k}(x)^{2} \geq \frac{\delta^{2} q}{8 k}\left\|x-x_{\bar{\Lambda}_{a}}\right\|^{2}
$$

Inserting this in the argument leading to (7.44), confirms the claim.

An extreme case where the algorithm would not perform well is $x_{i}=N^{-1 / 2}, i=$ $1, \ldots, N$, in which case $\sigma_{k}(x)=\sqrt{\frac{N-k}{N}}$ which stays close to one in the range of $k$ under consideration. Since the sets $\Lambda^{\prime}\left(r^{j}, \delta, k, \omega\right)$ are for most $\omega$ contained in the sets $\Lambda(x-$ $\left.x^{j-1}, \delta / 2, k\right)$, but $\left\|x-x^{j-1}\right\| \geq \sigma_{k}(x)$, no entry would actually satisfy $\left|x_{i}\right|=N^{-1 / 2} \geq$ $\frac{\delta}{2 \sqrt{k}}\left\|x-x^{j-1}\right\|$ so that the sets $\bar{\Lambda}_{j}$ would not build up. On the other hand, in such a case it would be irrelevant which entries to pick. 


\section{Appendix: random matrices satisfying $\mathrm{P} 1$ and $\mathrm{P} 2$}

In this section, we prove the validity of $\mathbf{P} \mathbf{1}$ and $\mathbf{P} \mathbf{2}$ for three standard examples of random matrices:

(i) Gaussian: the entries $\Phi_{i, j}$ are i.i.d. centered Gaussian variables of variance $1 / n$.

(ii) Bernoulli: the entries $\Phi_{i, j}$ are i.i.d. Bernoulli variables with values $\pm 1 / \sqrt{n}$.

(iii) Unit vectors: the columns $\phi_{i}$ are i.i.d. under the uniform law on the $n$-dimensional sphere.

We also show that $\mathbf{P} \mathbf{1}$ implies RIP.

\subsection{Proof of P1}

By linearity it is sufficient to consider a vector $x$ of norm 1 and therefore evaluate $\operatorname{Prob}\left\{\left|\|\Phi x\|^{2}-1\right| \geq \delta\right\}$.

The validity of $\mathbf{P} \mathbf{1}$ for Gaussian and Bernoulli matrices is a consequence of Lemma 6.1 of [12], which establishes this property for a more general class of random matrices with i.i.d. entries that have a subgaussian distribution.

For matrices consisting of random unit vectors, we notice that the function

$$
M\left(\phi_{1}, \cdots, \phi_{n}\right)=\left\|\sum_{j=1}^{N} x_{j} \phi_{j}\right\|=\|\Phi x\|
$$

is a Lip 1 function from $\left(\mathbb{R}^{\mathrm{n}}\right)^{\mathrm{N}}$ to $\mathbb{R}$ since

$$
\left|M\left(\phi_{1}, \cdots, \phi_{n}\right)-M\left(\phi_{1}^{\prime}, \cdots, \phi_{n}^{\prime}\right)\right| \leq\left\|\sum_{j=1}^{N} x_{j}\left(\phi_{j}-\phi_{j}^{\prime}\right)\right\| \leq\left(\sum_{j=1}^{N}\left\|\phi_{j}-\phi_{j}^{\prime}\right\|^{2}\right)^{1 / 2}
$$

The uniform product measure on an $N$-fold tensor product of $S_{n-1}$ by itself has the same concentration function of the form $e^{-(n-1) \delta^{2} / 2}$ as $S_{n-1}$ (see [18]) and therefore, using the notation $X=\|\Phi x\|$

$$
\operatorname{Prob}(|X-\mu| \geq \delta) \leq 2 e^{-(n-1) \delta^{2} / 2}
$$

where $\mu$ is the median of $X$. It is easy to derive from this a similar estimation where the median $\mu$ is replaced by the average $E(X)$ : assume that $X$ is a random variable that satisfies

$$
\operatorname{Prob}(|X-\mu| \geq \delta) \leq a e^{-b \delta^{2}}
$$

with $a \geq 1$. Then, assuming without loss of generality that $E(X) \geq \mu$, we obtain

$$
E(X)-\mu \leq \int_{0}^{+\infty} a e^{-b \delta^{2}}=\frac{a}{2} \sqrt{\frac{\pi}{b}}
$$


It follows that $\operatorname{Prob}(|X-E(X)| \geq \delta) \leq \operatorname{Prob}\left(|X-\mu| \geq \frac{\delta}{2}\right)$ when $\frac{a}{2} \sqrt{\frac{\pi}{b}} \leq \frac{\delta}{2}$. Since $\operatorname{Prob}(|X-E(X)| \geq \delta) \leq 1$, we have for any $\delta \geq 0$,

$$
\operatorname{Prob}(|X-E(X)| \geq \delta) \leq \tilde{a} e^{-b \delta^{2} / 4}, \quad \tilde{a}=e^{\pi a^{2} / 4},
$$

where we have used that $a \geq 1$. In the present case $X=\|\Phi x\|$, this gives

$$
\operatorname{Prob}(|X-E(X)| \geq \delta) \leq e^{\pi} e^{-(n-1) \delta^{2} / 4} \leq 27 e^{-(n-1) \delta^{2} / 4}
$$

By integration, we obtain

$$
E\left(|X-E(X)|^{2}\right) \leq 54 \int_{0}^{+\infty} t e^{-(n-1) t^{2} / 4} d t=\frac{108}{n-1}
$$

Since $E\left(\|\Phi x\|^{2}\right)=\|x\|^{2}=1$, we thus have

$$
0 \leq 1-E(X)^{2}=E\left(X^{2}\right)-E(X)^{2}=E\left(|X-E(X)|^{2}\right) \leq \frac{108}{n-1},
$$

which implies

$$
0 \leq 1-E(X) \leq \frac{108}{n-1}
$$

It follows from (8.1) and (8.2) that when $\delta \geq \frac{216}{n-1}$

$$
\operatorname{Prob}(|X-1| \geq \delta) \leq \operatorname{Prob}(|X-E(X)| \geq \delta / 2) \leq 27 e^{-(n-1) \delta^{2} / 16}
$$

On the other hand, if $\delta \leq \frac{216}{n-1}$, we have

$$
\operatorname{Prob}(|X-1| \geq \delta) \leq 1 \leq e^{\frac{2916}{n-1}} e^{-(n-1) \delta^{2} / 16}
$$

Therefore, in all cases we have

$$
\operatorname{Prob}(|X-1| \geq \delta) \leq C_{0} e^{-(n-1) \delta^{2} / 16},
$$

with $C_{0}=\max \left\{27, e^{\frac{2916}{n-1}}\right\}$. We conclude by

$$
\begin{aligned}
\operatorname{Prob}\left(\left|\|\Phi x\|^{2}-1\right| \geq \delta\right) & =\operatorname{Prob}\left(\left|X^{2}-1\right| \geq \delta\right) \\
& \leq \operatorname{Prob}(|X-1| \geq \delta / 3)+\operatorname{Prob}(|X+1| \geq 3) \\
& \leq \operatorname{Prob}(|X-1| \geq \delta / 3)+\operatorname{Prob}(|X-1| \geq 1) \\
& \leq 2 C_{0} e^{-(n-1) \delta^{2} / 144}
\end{aligned}
$$

for all $0 \leq \delta \leq 1$, which shows that $\mathbf{P} \mathbf{1}$ holds. 


\subsection{Proof of P2}

Again, by linearity, it is sufficient to consider a vector $z$ of norm 1 and evaluate $\operatorname{Prob}\left\{\left|\left\langle z, \phi_{l}\right\rangle\right| \geq\right.$ $\delta\}$.

For Gaussian matrices, we note that $\left\langle z, \phi_{l}\right\rangle=\sum_{i=1}^{n} z_{i} \Phi_{i, l}$ is a centered Gaussian variable with variance $1 / n$. From this it follows that

$$
\operatorname{Prob}\left\{\left|\left\langle z, \phi_{l}\right\rangle\right| \geq \delta\right\}=\frac{2}{\sqrt{2 \pi}} \int_{t \geq \sqrt{n} \delta} e^{-t^{2} / 2} d t \leq \frac{2}{\sqrt{2 \pi}} e^{-n \delta^{2} / 2} \int_{t \geq \sqrt{n} \delta} e^{-(t-\delta \sqrt{n})^{2} / 2} d t=e^{-n \delta^{2} / 2} .
$$

Therefore P2 holds with $b_{2}=1$ and $c_{2}=1 / 2$.

For Bernoulli matrices, we invoke Hoeffding's inequality [16], which states that for a sequence of independent variables $v_{i}$ with mean zero and such that $\left|v_{i}\right| \leq m_{i}$ almost surely, one has

$$
\operatorname{Prob}\left\{\left|\sum v_{i}\right| \geq \delta\right\} \leq 2 e^{-\frac{\delta^{2}}{2 \sum m_{i}^{2}}}
$$

It follows that

$$
\operatorname{Prob}\left\{\left|\left\langle z, \phi_{l}\right\rangle\right| \geq \delta\right\} \leq 2 e^{-\frac{\delta^{2}}{2 \sum z_{i}^{2} / n}}=2 e^{-n \delta^{2} / 2} .
$$

Therefore $\mathbf{P} 2$ holds with $b_{2}=2$ and $c_{2}=1 / 2$.

For matrices consisting of random unit vectors, $\operatorname{Prob}\left\{\left|\left\langle z, \phi_{l}\right\rangle\right| \geq \delta\right\}$ is the ratio between the measure of the set $S_{\epsilon, n-1}:=\left\{x \in S_{n-1},|\langle z, x\rangle| \geq \delta\right\}$ and the measure of the whole sphere $S_{n-1}$. It is well known that this ratio tends exponentially to 0 as $n \rightarrow+\infty$. More precisely, using that the uniform measure on $S_{n-1}$ has a concentration function of the form $e^{-(n-1) \delta^{2} / 2}$ (see [17]), one obtains that

$$
\operatorname{Prob}\left\{\left|\left\langle z, \phi_{l}\right\rangle\right| \geq \delta\right\} \leq 2 e^{-(n-1) \delta^{2} / 2} .
$$

Since, the inner product is clearly $\leq 1$, we only need to consider $\delta \leq 1$, in which case we find that $\mathbf{P 2}$ holds with $b_{2}=2$ and $c_{2}=1 / 4$ provided $n \geq 2$.

\subsection{P1 implies RIP}

The restricted isometry property RIP, introduced by Candes, Tao and Romberg [8, 9] states that $\Phi$ acts close to an isometry on all $m$ sparse vector. In other words, for some $0<\eta<1$

$$
(1-\eta)\left\|x_{T}\right\| \leq\left\|\Phi x_{T}\right\| \leq(1+\eta)\left\|x_{T}\right\|, \quad x \in \mathbb{R}^{N},|T| \leq k .
$$

Following the approach in [3], we shall prove that P1 implies that (2.3) holds with probability larger than $1-b_{1} e^{-n \frac{c_{1} \eta^{2}}{4}+m[\log (\eta N / m)+\log (12 / \eta)]}$.

We first fix $T$ such that $|T| \leq N$ and consider the set $X_{T}$ of $N$-dimensional vectors with support contained in $T$. We shall prove that $\mathbf{P} \mathbf{1}$ implies the validity of

$$
(1-\eta)\|x\| \leq\|\Phi x\| \leq(1+\eta)\|x\|, \quad x \in X_{T},
$$


with probability larger than $1-b_{1}(12 / \eta)^{m} e^{-c_{1} n \eta^{2} / 4}$. Since the number of sets $T$ of cardinality $m$ is $\left(\begin{array}{c}N \\ m\end{array}\right) \leq(e N / m)^{m}$, it will follow from the union bound that $(2.3)$ holds with probability larger than $1-b_{1}(e N / m)^{m}(12 / \eta)^{m} e^{-c_{1} n \eta^{2} / 4}$, which is the announced result.

We choose a finite set $Q \subset X_{T}$ such that $\|q\|=1$ for all $q \in Q$ and such that for all $x \in X_{T}$ with $\|x\|=1$, there exists $q \in Q$ with $\|x-q\| \leq \eta / 4$. It is well known from covering numbers that one can choose such a set $Q$ with $|Q| \leq(12 / \eta)^{m}$ (see e.g. Chapter 13 of [19]).

Invoking $\mathbf{P} \mathbf{1}$ and a union bound, we thus obtain that with probability larger than $1-b_{1}(12 / \eta)^{m} e^{-c_{1} n \eta^{2} / 4}$, we have for all $q \in Q$.

$$
(1-\eta / 2)\|q\|^{2} \leq\|\Phi q\|^{2} \leq(1+\eta / 2)\|q\|^{2}
$$

which trivially gives

$$
(1-\eta / 2)\|q\| \leq\|\Phi q\| \leq(1+\eta / 2)\|q\|,
$$

Denoting by $M$ the norm of $\Phi$ restricted to $X_{T}$, we derive from the upper inequality and the covering property of $Q$,

$$
\begin{aligned}
M & =\sup _{x \in X_{T},\|x\| \leq 1}\|\Phi x\| \\
& \leq \sup _{x \in X_{T},\|x\| \leq 1} \inf _{q \in Q}(\|\Phi q\|+\|\Phi(x-q)\|) \\
& \leq 1+\eta / 2+M \sup _{x \in X_{T},\|x\| \leq 1}\|x-q\| \\
& \leq 1+\eta / 2+M \eta / 4 .
\end{aligned}
$$

It follows that $M \leq(1+\eta / 2) /(1-\eta / 4) \leq 1+\eta$ which gives the upper inequality in (8.8). The lower inequality follows from it since for all $x \in X_{T}$ with $\|x\|=1$ and $q \in Q$ such that $\|x-q\| \leq \eta / 4$, we have

$$
\|\Phi x\| \geq\|\Phi q\|-\|\Phi(x-q)\| \geq 1-\eta / 2-(1+\eta) \eta / 4 \geq 1-\eta .
$$

By linearity, the lower inequality is thus proved for any $x \in X_{T}$.

Acknowledgments: The authors wish to thank Sinan Gunturk and the Courant Institute of Mathematical Sciences who hosted us when much of this research was completed.

\section{References}

[1] D. Achlioptas, Database-friendly random projections, Proc. ACM SIGACTSIGMOD-SIGART Symp. on Principles of Database Systems (2001), pp. 274-281.

[2] A. Barron, A. Cohen, W. Dahmen and R. DeVore, Approximation and learning by greedy algorithms, Annals of Statistics 36 (2008), 64-94.

[3] R. Baraniuk, M. Davenport, R. DeVore, and M. Wakin, A simple proof of the restricted isometry property for random matrices Constructive Approximation, to appear in 2008.

[4] P. Boufounos, M. Duarte, and R. Baraniuk, Sparse signal reconstruction from noisy compressive measurements using cross validation; Proc. IEEE Workshop on Statistical Signal Processing, Madison, Wisconsin, August 2007. 
[5] S. Boucheron, G. Lugosi, and O. Bousquet, Concentration inequalities, Advanced Lectures in Machine Learning, 2004, Springer, pp. 208-240.

[6] A. Cohen, W. Dahmen and R. DeVore, Compressed sensing and best $k$-term approximation, submitted

[7] G. Cormode and S. Muthukrishnan, Towards an algorithmic theory of compressed sensing, Technical Report 2005-25, DIMACS, 2005.Graham Cormode, S. Muthukrishnan: Combinatorial Algorithms for Compressed Sensing. SIROCCO 2006: 280294.

[8] E. Candès and T. Tao, Decoding by linear programming, IEEE Trans. Inf. Theory, 51(2005), 4203-4215.

[9] E. Candès, J. Romberg, and T. Tao, Stable signal recovery from incomplete and inaccurate measurements, Comm. Pure and Appl. Math., 59(2006), 1207-1223.

[10] I. Daubechies, M. Defrise, C. De Mol, An iterative thresholding algorithm for linear inverse problems with a sparsity constraint, Communications on Pure and Applied Mathematics, 57(2004), 1413-1457.

[11] R. DeVore, I. Daubechies, M. Fornasier, and S. Güntürk, Iterative Re-weighted Least Squares, preprint.

[12] R. DeVore, G. Petrova and P. Wojtaszczyk, Instance-Optimality in Probability with an $\ell_{1}$-minimization decoder, preprint Texas A. \& M. University, 2008.

[13] R. DeVore and V. Temlyakov, Some remarks on greedy algorithms, Advances in Computational Mathematics 5, 173-187, 1996.

[14] D. Donoho, Compressed Sensing, EEE Trans. Information Theory, 52(2006), 12891306.

[15] J. A. Tropp and A. C. Gilbert, Signal recovery from random measurements via Orthogonal Matching Pursuit, IEEE Trans. Info. Theory, 53(2007), 4655-4666.

[16] W. Hoeffding, Probability inequalities for sums of bounded random variables, Journal of the American Statistical Association 58(1963), 13-30.

[17] M. Ledoux, The concentration of measure phenomenon, Mathematical Surveys and Monographs 89, American Mathematical Society, 2001.

[18] M. Ledoux, Isoperimetry and gaussian analysis, Lecture Notes in Math. 1648, 165294. Springer, 1996.

[19] G. G. Lorentz, M. von Golischek and Y. Makozov, Constructive approximation : advanced problems, vol. 304, Springer Grundlehren, Berlin, 1996.

[20] D. Needel and R. Vershynin, Uniform Uncertainty Principle and signal recovery via Regularized Orthogonal Matching Pursuit, Found. Comput. Math., to appear. 
[21] D. Needel and R. Vershynin, Signal Recovery from Inaccurate and Incomplete Measurements via Regularized Orthogonal Matching Pursuit, preprint, 2007.

[22] D. Needel and J. Tropp, CoSaMP: Iterative signal recovery from incomplete and inaccurate samples, preprint 2008.

[23] R. Ward, Cross validation in compressed sensing via the Johnson Lindenstrauss lemma, preprint, 2008.

[24] P. Wojtaszczyk, Instance optimality for $\ell_{1}$ minimization, preprint, 2007.

Albert Cohen, Laboratoire Jacques-Louis Lions, Université Pierre et Marie Curie 175, rue du Chevaleret, 75013 Paris, France, cohen@ann.jussieu.fr

Wolfgang Dahmen, Institut für Geometrie und Praktische Mathematik, RWTH Aachen, Templergraben 55, D-52056 Aachen Germany, dahmen@igpm.rwth-aachen,de

Ronald DeVore, Department of Mathematics, Texas A\&M University, College Station, TX, devore@math.tamu.edu 